\title{
In-Plane Photonic Crystal Devices using Fano Resonances
}

Bekele, Dagmawi; Yu, Yi; Yvind, Kresten; Mork, Jesper

Published in:

Laser and Photonics Reviews

Link to article, DOI:

10.1002/lpor.201900054

Publication date:

2019

Document Version

Peer reviewed version

Link back to DTU Orbit

Citation (APA):

Bekele, D., Yu, Y., Yvind, K., \& Mork, J. (2019). In-Plane Photonic Crystal Devices using Fano Resonances. Laser and Photonics Reviews, 13(12), [1900054]. https://doi.org/10.1002/lpor.201900054

\section{General rights}

Copyright and moral rights for the publications made accessible in the public portal are retained by the authors and/or other copyright owners and it is a condition of accessing publications that users recognise and abide by the legal requirements associated with these rights.

- Users may download and print one copy of any publication from the public portal for the purpose of private study or research.

- You may not further distribute the material or use it for any profit-making activity or commercial gain

- You may freely distribute the URL identifying the publication in the public portal

If you believe that this document breaches copyright please contact us providing details, and we will remove access to the work immediately and investigate your claim 
Review article

\title{
In-plane photonic crystal devices using Fano resonances
}

\author{
Dagmawi Bekele, Yi Yu, Kresten Yvind, and Jesper Mork* \\ DTU Fotonik, Technical University of Denmark, DK-2800, Kgs. Lyngby, Denmark \\ *jesm@fotonik.dtu.dk
}

DOI: https://doi.org/10.1002/lpor.201900054

\begin{abstract}
Nanocavity devices enabling concentration of light in a very small volume have resulted in several interesting applications over the past years. Of particular interest are the asymmetric resonance lineshapes known as Fano resonances, which result from the interference between a discrete mode of the nanocavity and a continuum of background modes. Compared to the conventional symmetric Lorentzian lineshape, asymmetric Fano lineshapes enable novel or improved device structures for use in optical switches, sensors, lasers, and narrow band filters. In this paper, we review the use of Fano lineshapes in photonic crystal membranes for realizing various optical signal processing functionalities. The basic theory of Fano resonances is presented, different photonic crystal Fano device geometries are reviewed, the nonlinear processes empowering the devices are explained, and an overview of all-optical signal processing demonstrations based on Fano resonances is given.
\end{abstract}

\section{Introduction}

Photonic cavities that strongly confine light have enabled realization of several types of devices, including semiconductor nanolasers,[1] nonlinear optical devices,[2] narrow-band filters,[3] and many more. The spontaneous emission rate of quantum dots, for example, can be enhanced inside a resonant cavity which is important for single photon sources [4] and low threshold lasers.[5] In nonlinear optics, cavities allow to achieve high optical intensities, which enable efficient light-matter interaction at low pulse energies.[6]

The resonance spectrum of an optical cavity is characterized by having a symmetriclineshape known as a Lorentzian resonance, peaking at the resonance frequency $\omega_{0}$ and with a linewidth $\Delta \omega$. The ratio $\omega_{0} / \Delta \omega$ is a dimensionless measure of the temporal storage capacity of the cavity and is denoted the quality factor, $Q$. The Lorentzian resonance is at the heart of 


\section{WILEY-VCH}

many photonics applications. However, another characteristic resonance lineshape emerges if one considers a narrow-band Lorentzian response superimposed on a broadband background field. The interference of these two contributions leads to a lineshape known as a Fano resonance, typically featuring an asymmetric frequency dependence.[7] It was Ugo Fano who in 1935 suggested the first theoretical explanation of the sharp asymmetric profiles observed in the absorption spectrum of a hydrogen atom by considering this interference phenomenon, which in that case is related to quantum interference of different transition paths.[8] Being a quite general wave phenomenon, similar sharp asymmetric profiles have been observed in various other physical systems, including photonics and plasmonics.[8]

For optical cavities much larger than the wavelength of light, the main loss channels determining the Q-factor are the desired out-coupling as well as absorption losses in the mirrors and waveguide. However, when the cavity size becomes comparable to the wavelength, scattering at abrupt interfaces leads to additional diffraction loss. It was shown in [9] that by "gently" confining light, such as not to introduce new components to the momentum vector of light that are not guided by the waveguide, the diffraction loss can be significantly reduced. In particular, photonic crystal structures have shown the ability to confine light within a cavity volume of the order of $(\lambda / 2 n)^{3}$, where $\lambda$ is the wavelength of light, and $n$ is the refractive index of the material.[10], [11] Achieving both high- $Q$ factor and small mode volume, $V$, is a main design goal for photonic cavities where one aims at realizing optical nonlinearities at small power levels. Recent design strategies even show the possibility of realizing cavities with strong sub-wavelength light confinement in dielectric structures.[12]-[14]

In this review, we focus on in-plane photonic crystal devices exploiting Fano resonances and the opportunities for using the characteristics of the Fano resonance to enable new optical signal processing functionalities. Note that reviews of Fano resonances in plasmonic and metamaterial and their applications in chemical and biological sensors can be found in Refs. [8], [15], [16]

\subsection{General characteristics of Fano resonances}

A Fano resonance arises due to the interference between a discrete mode and a continuum of modes, leading to characteristic lineshapes. In particular, asymmetric lineshape characterised 


\section{WILEY-VCH}

by having a transmission minimum and a transmission maximum in close spectral vicinity are often encountered, and this feature is beneficial for switching and modulation functionalities. The classical Fano transmission spectrum can be written in a general form as [8], [17]-[19]

$$
T(\delta)=\left|t_{B}\right|^{2} \frac{(q+\delta)^{2}}{1+\delta^{2}}
$$

where $t_{B}$ is the non-resonant amplitude transmission coefficient of the system, $q$ is the Fano asymmetry parameter or $q$-parameter, and the normalized frequency detuning is $\delta=\left(\omega-\omega_{0}\right) / \gamma$, where $\gamma$ is the cavity field decay rate given by $\gamma=\omega_{0} / 2 Q$. From Equation 1 we find that a transmission maximum of $T_{\max }=\left|t_{B}\right|^{2}\left(q^{2}+1\right)$ appears at $\delta=1 / q$, while a transmission minimum $T_{\min }=0$ appears at $\delta=-q .[8]$, [20]

The Fano asymmetry parameter determines the lineshape profile of the resonance. Figure 1 shows Fano transmission lineshape for four cases corresponding to $q$ values of $-1,0$, 1 , and 100. The case of $q=0$ (dashed black line) shows a symmetric dip at the resonance frequency indicating the occurrence of destructive interference between the discrete mode and the continuum of modes.

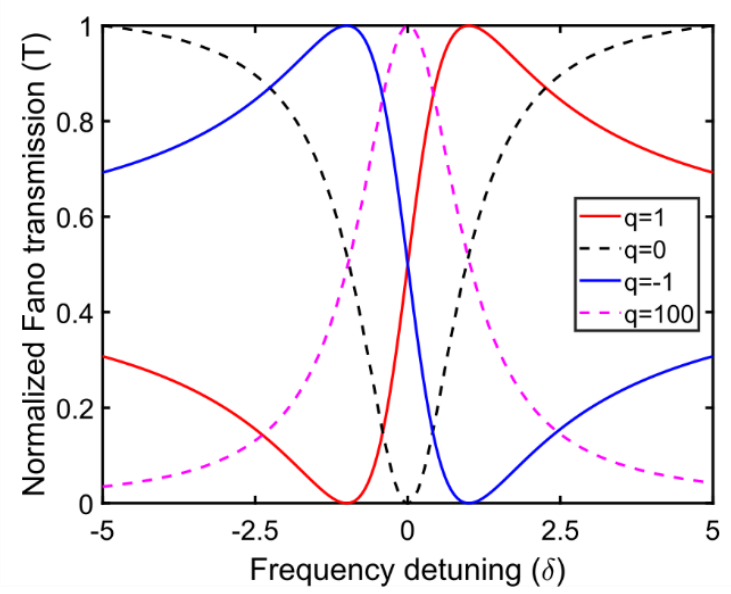

Figure 1: Fano resonance lineshapes for various values of the Fano asymmetry parameter. The dashed black line $(q=0)$ shows a symmetric dip at the resonance frequency. The solid blue (red) line shows an asymmetric lineshape with the transmission minimum spectrally located at higher (lower) frequency than the transmission maximum for $q=-1(1)$. The dashed purple line $(q=100)$ corresponds to the case where the interaction between the discrete mode and the continuum of modes is very weak, leading to a Lorentzian lineshape.

We refer to this as a symmetric Fano resonance, while in some literature it is denoted as an inverse Lorentzian or an antiresonance.[8] The cases of $q=-1$ and $q=1$, shown by solid 


\section{WILEY-VCH}

blue and solid red lines, respectively, correspond to asymmetric lineshapes, indicating the presence of both constructive and destructive interferences between the discrete mode and the continuum of modes. The spectral location of the transmission minimum is blue-shifted (redshifted) compared to the transmission maximum for $q=-1$ (1). Hence, the lineshapes are often referred to as blue-parity or red-parity Fano resonances for negative and positive values of the Fano asymmetry parameter, respectively.[8], [21], [22] In both cases, the resonance frequency is spectrally located midway between the transmission maximum and the transmission minimum. Note that by carefully choosing the Fano $q$-parameter, it is possible to design an asymmetric Fano resonance in which the resonance frequency of the cavity is spectrally located anywhere between the transmission maximum and transmission minimum. Finally, the dashed purple line is for the case of $q=100$. It shows a conventional Lorentzian lineshape at the cavity resonance with no features of interference between the discrete mode and the continuum of modes. The Lorentzian thus appears as a special case of the Fano resonance for $|q| \rightarrow \infty$.[8]

\subsection{Fano and Lorentzian resonances in photonic crystal devices}

Device structures for realizing Fano resonances can broadly be categorized as in-plane and out-of-plane configurations. In-plane structures usually involve coupling of one or more cavities to a waveguide which supports a continuum of modes. On the other hand, the out-ofplane configuration involves a discrete cavity mode or guided mode resonance of a slab structure interacting with an out-of-plane continuum of modes.[23] A comprehensive review of out-of-plane photonic crystal Fano resonances is provided in Ref. [23] In this section, we review some of the recent experimental demonstrations of in-plane photonic crystal configurations for realizing Fano resonances. Furthermore, a review of Lorentzian resonance structures is provided for ease of comparison between Fano and Lorentzian structures, which often appear similar to each other.

A number of devices realizing Fano and Lorentzian resonances using photonic crystal structures are presented in Figure 2 and Figure 3, respectively. The devices commonly consist of a nanocavity, which supports a discrete mode, and a line-defect photonic crystal waveguide or a bus waveguide that supports a continuum of modes. Nanocavities with strong spatial field confinement (low mode volume) and long temporal storage of the energy (high 


\section{WILEY-VCH}

$Q$-factor) lead to efficient light-matter interaction, thereby enabling devices with small footprint and low energy consumption.[24] In addition to the possibilities for realizing high-Q single mode or multimode nanocavities, photonic crystals $(\mathrm{PhC})$ allow implementation of waveguides and couplers with minimal change in the fabrication process.

Figure 2(a) region (i) shows a scanning electron microscope (SEM) image of a Fano structure consisting of a single mode input silicon waveguide connected to a multimode interference (MMI)-like section, a nanobeam cavity, and a subwavelength grating mode mixer.[25] The MMI-like section supports two transverse electric (TE) propagating modes. The width of the MMI section is designed in such a way that the difference between the effective refractive indices of the two TE waveguide modes is large. This allows the fundamental TE mode to interact strongly with the nanobeam cavity, creating a narrow-band transmission, while the first-order TE mode is insensitive to the periodic corrugation of the nanobeam, resulting in a flat-band transmission spectrum. The interference between these narrow-band and wide-band waveguide modes is facilitated by the subwavelength mode mixer, which results in two Fano resonances, i.e. one for each of the TE modes. A directional coupler is then used to couple-out the first order TE mode to a single mode output waveguide. The measured asymmetric Fano transmission spectrum together with a theoretical fit are shown in Figure 2(a) region (ii).

Figure 2(b) shows a silicon photonic crystal nanobeam cavity that is side-coupled to a bus waveguide with a Fabry-Perot (FP) resonator, region (i), and without FP resonator, region (ii).[26] The two holes in the bus waveguide act as partially reflecting elements forming a FP resonator of length $2 l$. Both structures give rise to Fano interference. However, the structure with the FP resonator in the bus waveguide helps to achieve a sharper asymmetric Fano lineshape with higher extinction ratio, as shown in Figure 2(b) region (iii) for a FP cavity length of $800 \mathrm{~nm}$.[27] Here, we would like to point out that the configuration with two airholes may not be treated as a simple FP cavity as the influence of evanescent Bloch modes for smaller FP cavities becomes large, leading to the breakdown of the single-mode description of the transmission of the system.[21] 


\section{WILEY-VCH}

(a)

(i)

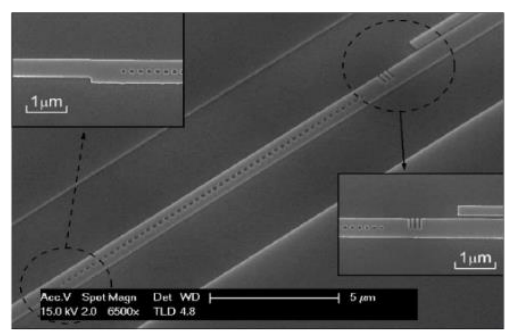

(ii)

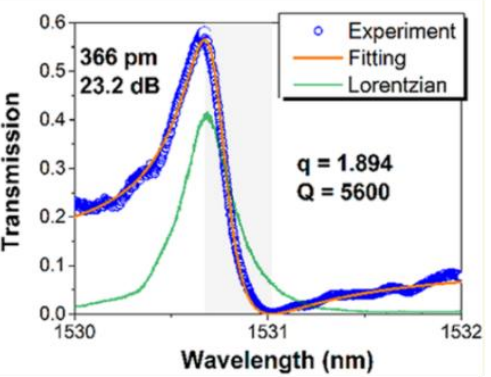

(c)

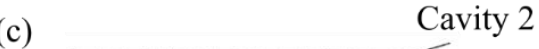

(e)
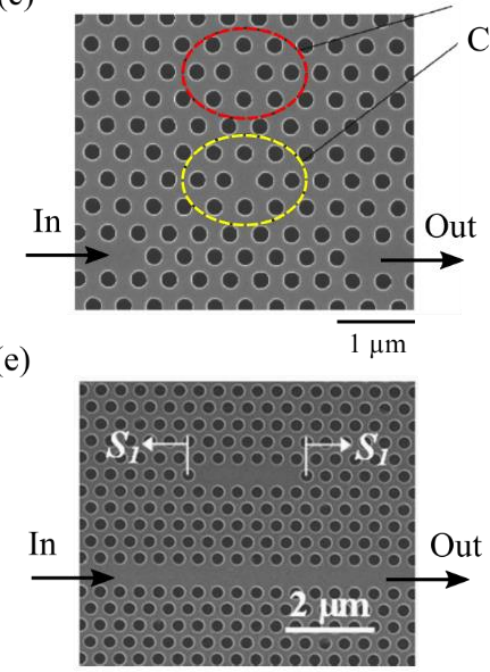

(b)

(i)

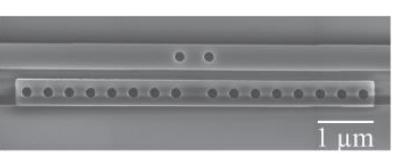

(ii)

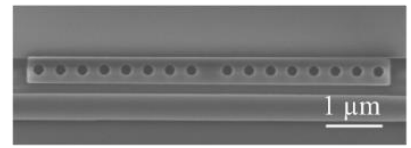

(iii)

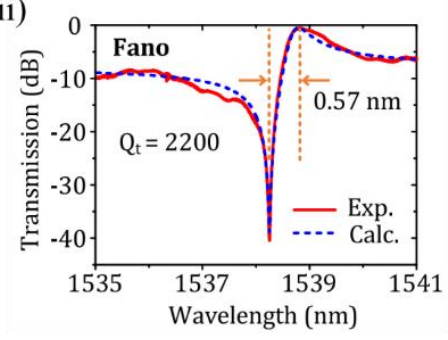

(d)

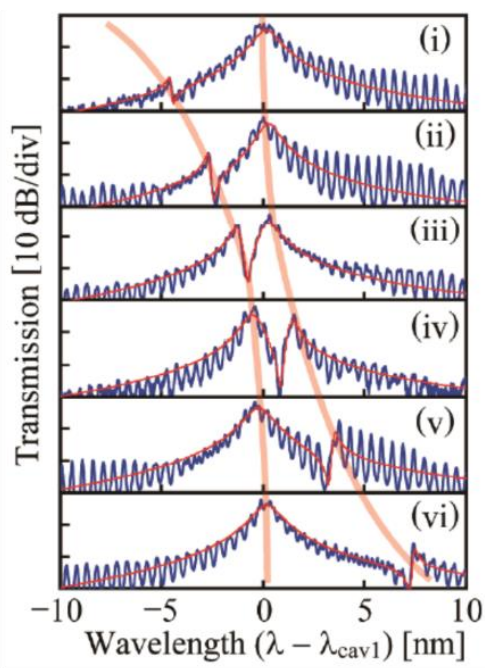

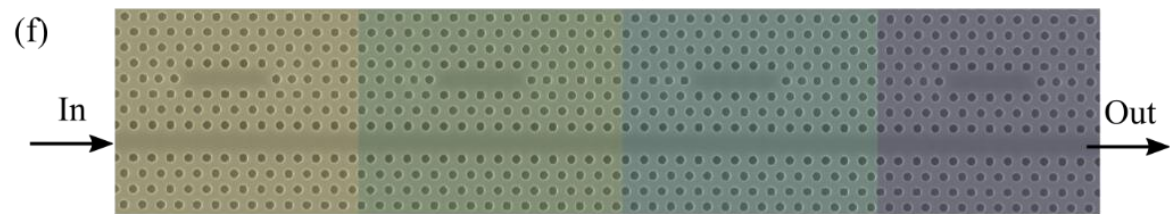

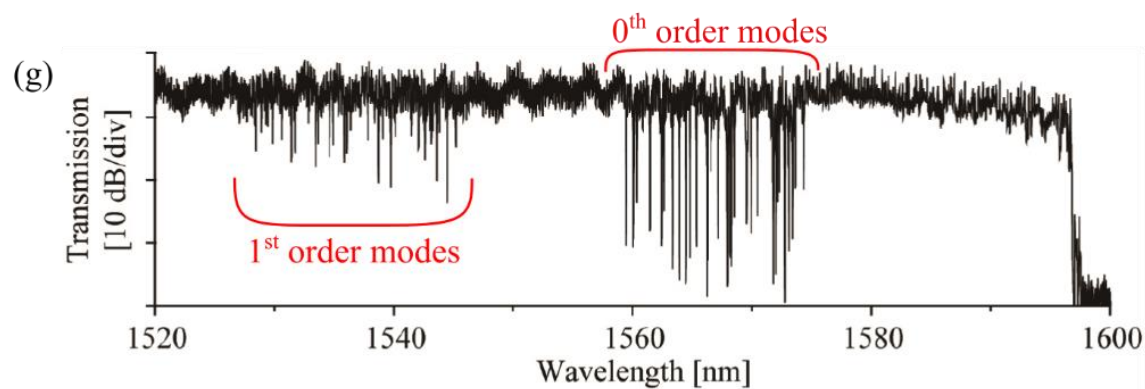

Figure 2: Various realizations of Fano resonances using photonic crystal platforms and their measured transmission spectra. (a) Fano structure consisting of an MMI-like input waveguide, a nanocavity, a 


\section{WILEY-VCH}

subwavelength mixer, and a directional coupler. (b) Fano structure consisting of nanobeam cavity side-coupled to a waveguide Fabry-Perot resonator. (c) Fano structure consisting of two coupled H0 nanocavities, input and output waveguides. (d) Transmission spectra of the structure in part (c) for different values of the detuning between the resonances of the two H0 cavities. (e) Fano structure consisting of L5 cavity side-coupled to a line-defect waveguide. (f) Fano structure consisting of four photonic crystal L4 cavities coupled to a single-mode waveguide. (g) Transmission spectrum of the device in part (f) consisting of 32 cavities, each supporting two modes, hence 64 dips in the transmission spectrum. (a) Reproduced with permission [25], Copyright 2018 American Chemical Society. (b) Part (i) and (ii) Reproduced with permission [26], Copyright 2013, AIP publishing. Part (iii) Reproduced with permission [27], Copyright 2018, The Optical Society. (c) and (d) Reproduced with permission [28], Copyright 2013, The Optical Society. (e) Reproduced with permission [29], Copyright 2007, AIP publishing. (f) and (g) Reproduced with permission [30], Copyright 2014, The Optical Society.

An InGaAsP photonic crystal membrane featuring two coupled H0 cavities is shown in Figure 2(c). Cavity 1 is coupled with line-defect waveguides for input and output of light. There are two optical paths created for the input light in the waveguide close to the resonance frequencies of the cavities. For the first path, light passes through both cavities and couples to the output waveguide. For the second path, light only passes through cavity 1 . The first path induces a larger phase change compared to the second optical path. Moreover, cavity 1 is designed to have low-Q factor, thus in essence acting as a mode continuum, while the high-Q mode of cavity 2 provides the discrete mode. The interference between these two paths results in an asymmetric Fano spectrum [28] as shown in Figure 2(d) for different values of the detuning between cavity 1 and cavity 2 . The detuning is varied by slightly shifting the inner most airholes of cavity 2.[28]

A Fano structure composed of a silicon photonic crystal waveguide side-coupled to an L5 cavity is shown in Figure 2(e). The innermost airholes of the cavity are shifted away from the cavity centre in order to achieve high Q-factors. Fano interference is observed as a result of the interference between the continuum of waveguide modes and the discrete mode of the cavity.[29] Several cavities, only four are shown here for clarity, can also be side-coupled to a single-mode photonic crystal waveguide as shown in Figure 2(f).[30] This Fano structure allows wavelength division multiplexing (WDM) functionalities by integrating up to 32 photonic crystal L4 cavities with slightly varying lattice constants while keeping the airhole radius constant.[30] Each of the L4 cavities supports two modes with free-spectral range (FSR) of around $30 \mathrm{~nm}$. The transmission spectrum of the device in Figure 2(f) is shown in 


\section{WILEY-VCH}

Figure 2(g).[30] It consists of 32 pairs of transmission dips, in which a single pair of dips corresponds to the $0^{\text {th }}$ and the $1^{\text {st }}$ order modes of each cavity.[30] Note that the resonance lineshape obtained from the transmission spectrum of the devices in Figure 2(e) and Figure 2(f) is a symmetric Fano lineshape. Similar to the structure shown in Figure 2(e), a GaAsbased photonic crystal L3 cavity side-coupled to a waveguide that exhibit Fano interference has also been reported.[31]

A GaAs photonic crystal structure consisting of an $\mathrm{H} 0$ cavity coupled to input and output waveguides is shown in Figure 3(a).[32] Light transmission from the input waveguide to the output waveguide is only possible around the resonance wavelength by tunnelling through the cavity, leading to a Lorentzian lineshape. The measured transmission spectrum with its Lorentzian fit, which is used to estimate the total Q-factor of 1200, is shown Figure 3(a) [right]. A similar device structure implemented in InGaAsP, having a total Q-factor of around 6500, is also reported in Ref.[6] Alternatively, instead of coupling to the through-port, crossport coupling is also possible, in which case a total Q-factor of 4100 was obtained, c.f. Figure 3(b).[33]-[35] 


\section{WILEY-VCH}
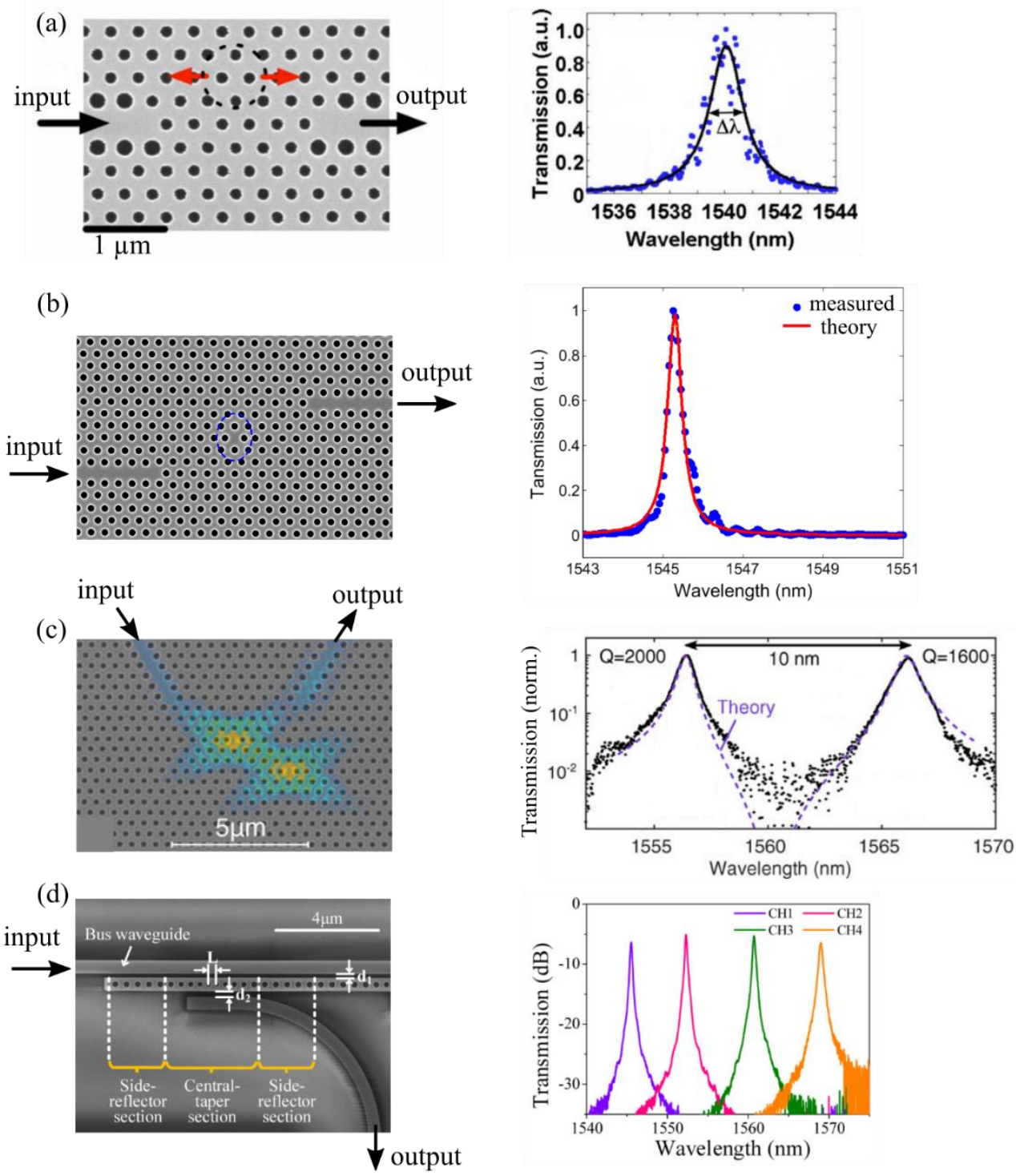

Figure 3: Various realizations of Lorentzian resonances using photonic crystal structures and their measured transmission spectra. The device geometry consists of an $\mathrm{H} 0$ cavity coupled to input and output waveguides in (a) GaAs or (b) InP. (c) Lorentzian structure consisting of two identical H0 cavities coupled together to form a photonic molecule coupled to the input and output waveguides. (d) Lorentzian structure consisting of an input bus waveguide, photonic crystal nanobeam cavity, and output waveguide. (a) Reproduced with permission [32], Copyright 2009, AIP Publishing. (b) (left) Reproduced with permission [34], Copyright 2014, AIP Publishing. (right) Reproduced with permission [33], Copyright 2013, The Optical Society. (c) Reproduced with permission [36], Copyright 2016, Wiley-VCH. (d) Reproduced with permission [37], Copyright 2018, The Optical Society.

A Lorentzian structure composed of two identical $\mathrm{H} 0$ cavities, which are coupled to each other forming a photonic molecule, and connected to input and output waveguides based on 


\section{WILEY-VCH}

GaAs material is shown in Figure 3(c).[36] Similar to other Lorentzian structures, light transmission from the input port to the output port is only possible via the photonic molecule. The coupling between the two cavities results in the splitting of the resonance into two peaks which are separated by $10 \mathrm{~nm}$ [36], c.f. Figure 3(c) [right]. The theoretical fit to the measured spectrum using coupled mode theory shows that the total Q-factor of the two resonances are 1600 and 2000. This structure, similar to the structure in Figure 2(f), allows larger spectral separation between a pump and a probe signal for use as an all-optical switch, c.f. Section 3.

Another type of Lorentzian structure, composed of an input bus waveguide side-coupled to a nanobeam cavity and an output waveguide, is shown in Figure 3(d). [37] By extending the nanobeam to form a series of nanocavities of different resonance frequencies, a multichannel device with WDM functionality has been realized on a silicon platform.[37] The transmission spectra of four such channels are shown in Figure 3(d)[right].

\subsection{Fano resonance in a waveguide-coupled cavity structure}

Hence on we consider the generic system of a waveguide that is side-coupled to a cavity, cf. Figure 4. Light can be coupled in and out of the structure through ports 1 and 2 of the waveguide. The energy in the cavity can decay into port 1 and port 2 with decay rates $\gamma_{1}$ and $\gamma_{2}$, respectively. The waveguide contains a partially transmitting element (PTE), which allows to control the asymmetry of Fano resonance through its amplitude transmission coefficient, $t_{B}$, as proposed in Ref.[38] A coupled mode theory (CMT) model for this waveguide-coupled cavity system is discussed below based on Ref. [8], [17], [20], [33], [38], [39]

The field amplitude in the waveguide is $s_{y}^{x}(t)$, with $x=i$ or $x=o$ representing the signal field amplitude at the input or output ports, respectively. The forward and the backward propagating fields are denoted by $y=+$ and $y=-$, respectively. The cavity has a resonance frequency of $\omega_{o}$, and its field amplitude is denoted by $a(t)$. Additionally, the intrinsic loss of the cavity is represented by $\gamma_{i n}$ which is related to the intrinsic quality $\left(Q_{i n}\right)$ factor of the cavity as $\gamma_{i n}=\omega_{o} /\left(2 Q_{i n}\right)$. Here, the total cavity field decay rate $\left(\gamma_{t}\right)$ is related to the total quality $\left(Q_{t}\right)$ as $\gamma_{t}=\omega_{o} /\left(2 Q_{t}\right)$, where $\gamma_{t}=\gamma_{1}+\gamma_{2}+\gamma_{i n}$. 


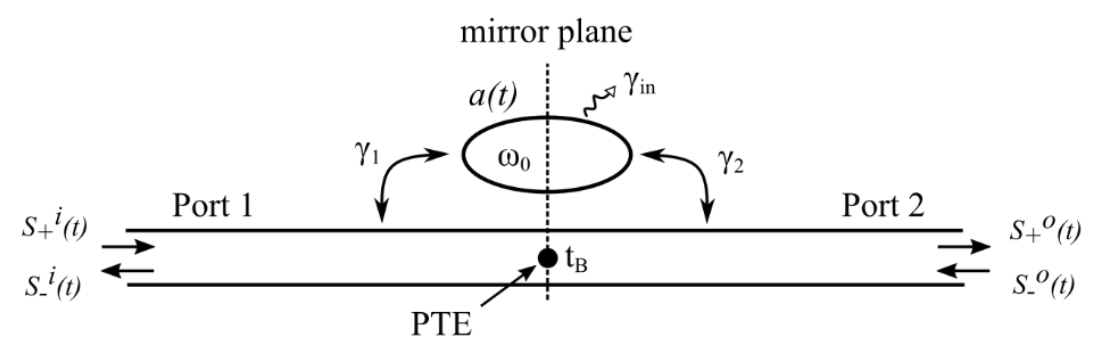

Figure 4: Coupled-mode theory (CMT) model of coupled waveguide-cavity system.

The structure can be excited with light of frequency $\omega_{s}$ coupled to the input port (port 1) while setting the power from the right to zero, i.e. $s_{-}^{o}(t)=0$. In this case, the rate of change of the cavity field can be written as

$$
\dot{a}(t)=\left(-i\left(\omega_{o}-\omega_{s}\right)-\gamma_{1}-\gamma_{2}-\gamma_{i n}\right) a(t)+\sqrt{2 \gamma_{1}} e^{i \theta_{1}} s_{+}^{i}(t),
$$

where $e^{i \theta_{1}}$ is the phase of the coupling between the input port and the cavity. The first term in Equation 2 accounts for the detuning-dependence of the amplitude of the cavity field envelope, and its decay via the various decay rates, while the second term represents the feeding of the cavity field as a result of the coupling to the input field.

Similarly, the output field amplitude of the structure can be written as

$$
s_{+}^{o}(t)=-i t_{B} s_{+}^{i}(t)+\sqrt{2 \gamma_{2}} e^{i \theta_{2}} a(t),
$$

where $e^{i \theta_{2}}$ is the phase of the coupling between the output port and the cavity. The first term in Equation 3 represents the direct transmission of light to the output port (port 2). The amplitude of this direct transmission path is controlled by the amplitude transmission coefficient, $t_{B}$, of the PTE placed in the middle of the waveguide. The second term in Equation 3 represents the contribution of the cavity field that couples to the output port. It is the interference of the two terms in Equation 3 that gives rise to the Fano resonance lineshape.

Finally, the transfer function of the cavity-waveguide coupled structure is calculated using $t(\omega)=s_{+}^{o}(t) / s_{+}^{i}(t)$, where the power transmission of the system is given by

$$
T(\omega)=|t(\omega)|^{2}=\left|-i t_{B}+\frac{2 \sqrt{\gamma_{1} \gamma_{2}} e^{i\left(\theta_{1}+\theta_{2}\right)}}{\gamma_{1}+\gamma_{2}+\gamma_{i n}+i\left(\omega_{o}-\omega\right)}\right|^{2},
$$

where the phase term $e^{i\left(\theta_{1}+\theta_{2}\right)}$ can be calculated from the values of $t_{B}, \gamma_{1}, \gamma_{2}$ using the requirements of energy conservation and time-reversal symmetry as [40] 


\section{WILEY-VCH}

$$
e^{i\left(\theta_{1}+\theta_{2}\right)}=i t_{B} \sqrt{\frac{\gamma_{2}}{\gamma_{1}}}\left[\frac{1}{r_{B} e^{-i P 2 \theta_{1}+1}}\right] .
$$

Here, the factor $P$ takes the value $+1(-1)$ for a cavity mode that is even (odd) with respect to the mirror symmetry plane, cf. Figure 4, passing through the center of the cavity, and $\cos \left(2 \theta_{1}\right)=\frac{\left(\gamma_{2}-\gamma_{1}\right) t_{B}^{2}-2 \gamma_{1} r_{B}^{2}}{2 \gamma_{1} r_{B}}$. The amplitude transmission and reflection coefficients of the PTE are related by $t_{B}^{2}+r_{B}^{2}=1$.

Comparing Equation 4 with the classical Fano formula of Equation 1 for the lossless case, i.e. $\gamma_{i n}=0$, the Fano asymmetry parameter can be written as [17], [20]

$$
q=-\tan (\Delta) \text {, }
$$

where $\Delta$ is the phase difference between the resonant and non-resonant pathways at the cavity resonance frequency. It is related to the transmission coefficient and the cavity decay rates as $\Delta=P \cos ^{-1}\left(\frac{-\left|t_{B}\right|\left(\gamma_{1}+\gamma_{2}\right)}{2 \sqrt{\gamma_{1} \gamma_{2}}}\right)$, where the factor $P$, similar to Equation 5 , takes the value $+1(-1)$ for even (odd) cavity modes.[20] Here, it should be noted that one can design a Fano structure with desired phase relationship between the discrete mode and mode continuum by choosing the magnitude of $t_{B}$, the coupling rates, $\gamma_{1}$ and $\gamma_{2}$, and the symmetry of the cavity mode, $P$. However, this would require significant change in the design of the waveguide-coupled cavity structure. In section 1.4, we discuss a rather simpler approach to change the phase relationship, $\Delta$, without the need of re-designing the cavity.

The spectral separation between the transmission minimum and maximum of the asymmetric Fano resonance for mirror symmetric structure can be written as $\Delta \omega=\gamma / t_{B} r_{B}$, where $\gamma_{1}=\gamma_{2}=\gamma / 2$.[41] For simplicity of calculation, the out-of-plane decay rate from the cavity is neglected, assuming strong coupling between the cavity and the waveguide. The minimum extrema separation of $2 \gamma$ is then achieved when $t_{B}=1 / \sqrt{2}$. Therefore, the smallest spectral separation between the transmission maximum and minimum of an asymmetric Fano resonance is equal to the linewidth of the cavity.[41]

Figure 5 shows SEM images of fabricated indium phosphide (InP) photonic crystal membrane structures and their corresponding measured transmission spectra. The structures consist of hexagonally arranged airholes incorporating nanocavities, indicated by dotted blue lines, and line defect waveguides. The nanocavities shown in Figure 5(a) and Figure 5(d) are 


\section{WILEY-VCH}

quasi-H1 type, while in Figure 5(b) and Figure 5(c) they are of H0 type.[42] The quasi-H1 type is formed by reducing the radius of the center airhole and shifting the airholes around the cavity away from the cavity center. The H0 type is formed by shifting the airholes around the cavity away from the cavity center. The line-defect waveguides are of W1-type formed by removing one row of airholes and shifting the inner most row of airholes towards the waveguide center.[43], [44] Assuming the same $Q$-factor and cavity mode symmetry, the choice of quasi-H1 or $\mathrm{H} 0$ does not affect the lineshapes. However, an H0 cavity features larger $Q / V$, and better overlap of the cavity mode with the material resulting in efficient lightmatter interaction.[43]

The transmission spectrum of the structure in Figure 5(a) shows a dip indicating the occurrence of destructive interference between the discrete mode of the cavity and the continuum of waveguide modes. This is a symmetric Fano resonance. Based on the CMT fit (red line) to the measured spectrum (black line), the total Q factor of the cavity is estimated to be around 10400.[44]

(a) symmetric Fano
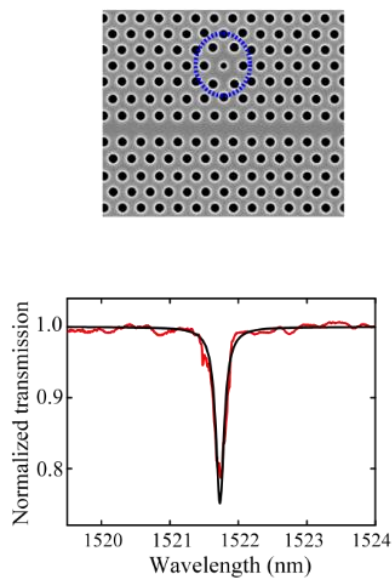

(b) red-parity Fano
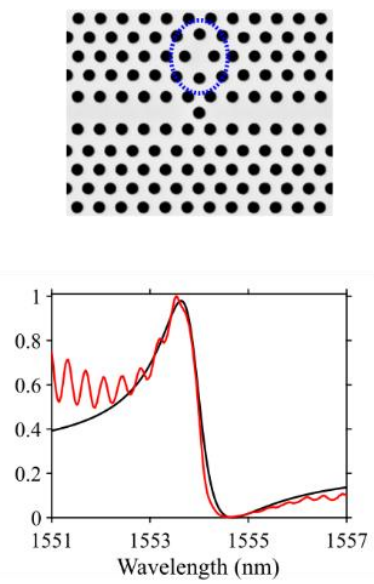

(c) blue-parity Fano
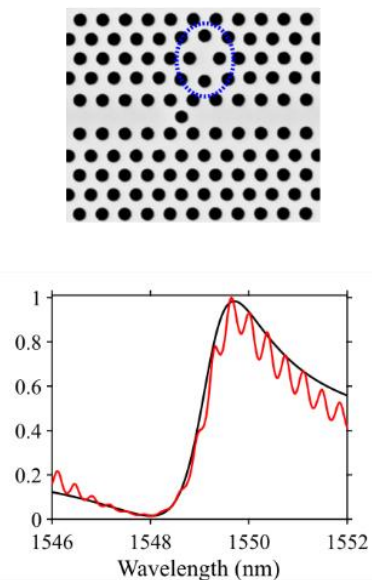

(d) Lorentzian
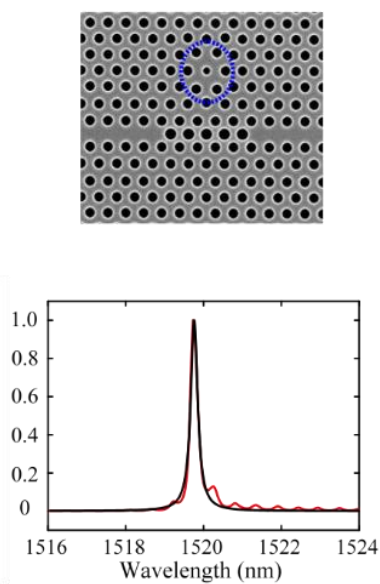

Figure 5: SEM images of fabricated photonic crystal cavity-waveguide coupled structures and their measured transmission spectra (red lines), including CMT fits to the measurements (black lines). (a) symmetric Fano structure and its lineshape. (b) red-parity asymmetric Fano resonance. The PTE airhole is placed in the mid-plane passing through the center of the nanocavity.[41] (c) blue-parity asymmetric Fano resonance. The PTE airhole is placed one lattice constant to the left of the nanocavity symmetry plane.[41] (d) Lorentzian structure and its lineshape. The transmission of light through the line-defect waveguide is blocked by the use of 5 large airholes placed in the middle of the waveguide. (a) and (d) Reproduced with permission [44], Copyright 2014, AIP publishing. 


\section{WILEY-VCH}

The transmission spectra of the structures in Figure 5(b) and Figure 5(c) indicate redparity and blue-parity Fano resonances, respectively. For the red-parity device, the PTE airhole is placed in the waveguide at the mid-plane passing through the center of the nanocavity while for the blue-parity device, the PTE airhole is placed one lattice constant to the left of the mid-plane. This breaks the mirror symmetry of the device, i.e. $\gamma_{1} / \gamma_{2} \neq 1$. Therefore, the phase term of Equation 5 can be varied via the radius and the position of the PTE airhole.[21] The structure in Figure 5(d) is a Lorentzian structure in which the transmission of light through the line-defect waveguide is completely blocked by the use of 5 large airholes placed in the middle of the waveguide. However, for input wavelengths coinciding with the resonance wavelength of the cavity, light transmission to the output port is made possible by tunnelling through the cavity.[44]

So far, we have discussed the key features of the lineshapes of Fano and Lorentzian resonances. Here, we illustrate the main advantage of the use of asymmetric Fano resonance over that of the Lorentzian resonance for realizing active device functionalities, specifically considering optical switching. Figure 6 shows the transmission spectra of Lorentzian and Fano resonances of an unshifted cavity, solid black line, and a shifted cavity, dashed blue line. The blue shaded regions represent the spectral location of the input signal that is to be switched. The initial spectral position of the resonance, shown by the black solid line, implies that the input signal experiences the minimum transmission through the device, hence representing the switch-off state. On the other hand, the shifted cavity transmission spectrum corresponds to maximum transmission of the input signal through the device, thus representing the switch on-state. Both resonances have the same total Q factor of around 1700. The main difference here is that the Lorentzian resonance requires a larger spectral shift than the Fano resonance in order to achieve a similar level of signal suppression, i.e. $\Delta \lambda_{\mathrm{L}}>\Delta \lambda_{\mathrm{F}}$. This implies that the Fano resonance has a significant advantage by reducing the energy required to shift the resonance, making it suitable for realizing energy-efficient integrated optical switches. In the following section, we discuss the physical mechanisms employed to achieve efficient resonance shifts. 


\section{WILEY-VCH}
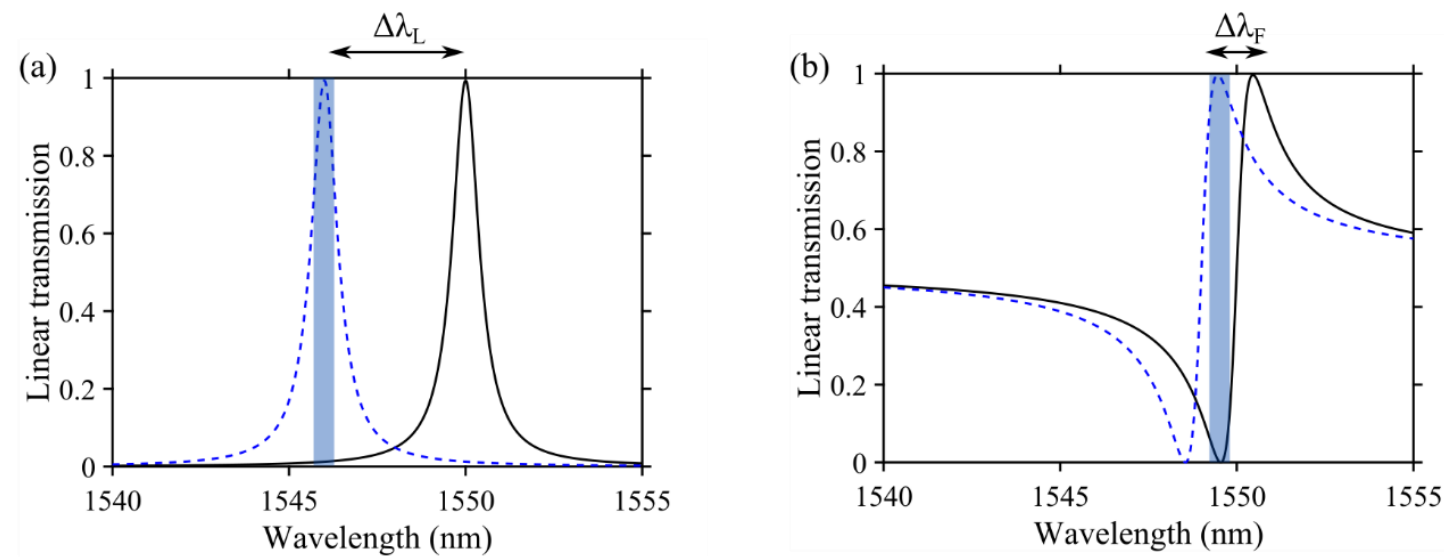

Figure 6: Comparison of optical switching using (a) Lorentzian resonance and (b) asymmetric Fano resonance. The blue shaded regions represent the input signal spectral location. The unshifted resonances are shown by the black solid lines, leading to minimum transmission of the input signal (off-state). The dashed blue lines indicate the shifted resonance spectra. In this case, the transmission of the input signal through the device is strongly enhanced, representing the switch on-state.

\section{Nonlinear and dynamical characteristics of the nanocavity}

As illustrated in Figure 6, optical switching can be achieved by shifting the resonance frequency, which is possible by locally changing the refractive index of the cavity. Two of the most commonly used nonlinear processes for achieving resonance shifts in nanocavities are Kerr-based refractive index changes, and carrier-induced refractive index changes due to twophoton absorption (TPA). Generally, Kerr-based nonlinearities are preferred due to the instantaneous nature of the refractive index changes as a function of the intensity of light which potentially paves the way for realization of ultra-fast devices. However, commonly used semiconductor materials such as $\mathrm{Si}, \mathrm{GaAs}$, and $\mathrm{InP}$ at telecom wavelengths have relatively large TPA coefficients, which in many cases dominate the corresponding Kerr coefficients. Due to the strong field concentration in nanocavities, TPA provides an efficient mechanism for free-carrier generation, and the corresponding refractive index change can be used to realize efficient all-optical switches. In this section, we discuss the free-carrier induced refractive index changes, and the carrier dynamics of InP based photonic crystal nanocavities based on Ref. [31], [33], [45], [46]

The highly localized intensity in an $\mathrm{InP} \mathrm{PhC}$ nanocavity with bandgap energy, $E_{g}$, of around $1.34 \mathrm{eV}$ and operating at telecom wavelengths of around $0.8 \mathrm{eV}$ allows the generation 


\section{WILEY-VCH}

of free-carriers via a two-photon absorption process. The density of these free-carriers, $N$, generated by TPA is given by [47]

$$
N=\tau \frac{\beta_{T P A} I^{2}}{2 \hbar \omega}
$$

where $\beta_{T P A}$ is the TPA coefficient, $I$ is the intensity of light, $\hbar \omega$ is the energy of a photon, and $\tau$ is the carrier recombination lifetime. These accumulated free-carriers give rise to a change in the complex refractive index via three nonlinear processes namely band-filling effects, band-gap shrinkage and free-carrier absorption or plasma dispersion.[46]

A decrease in absorption for photon energies slightly larger than the fundamental bandgap, $E_{g}$, can be observed when a large density of free-carriers is present. This is because the lowest energy states in the conduction band are already filled and hence electrons from the valence band require energies greater than $E_{g}$ to be optically excited into the conduction band. This decrease in the absorption coefficient results in reduction of the refractive index of the material, and the phenomena is known as the band-filling effect.[46]

Another nonlinear process associated with free-carriers is bandgap shrinkage. With the lower energy states occupied at the conduction band, electrons repel each other by Coulomb forces resulting in screening of electrons and hence lowering the bandgap energy.[46] This results in a slight increase in the absorption coefficient leading to an increase in the refractive index of the material. At telecom wavelengths and room temperature, the effect on the refractive index from bandgap shrinkage in InP appears to be negligible.[45], [46]

A free electron can also absorb a photon and move to a higher energy state within the conduction band. This intraband free-carrier absorption can be described using the Drude model which gives the change in the refractive index change as

$$
\Delta n=-\frac{e^{2}}{2 m_{e} \epsilon_{0} n \omega_{s}^{2}} N,
$$

where $e$ is the elementary electric charge, $m_{e}$ is the effective mass of the electron, and $\omega_{s}$ is the angular frequency of the light. This change in refractive index due to free-electron is also known as plasma dispersion. Note that the sign of $\Delta n$ is negative and it is proportional to the free-carriers concentration.

The carriers generated by TPA have an important adverse effect of increasing the temperature of the device, since a significant fraction of this absorbed power is converted to 


\section{WILEY-VCH}

kinetic energy of electrons leading to heating.[31], [33], [48] The change in the refractive index due to a change in the temperature is given as $n=n_{0}+n_{T} \Delta T$, where the quantity $n_{T}=$ $d n / d T$ describes the temperature dependence of the refractive index. For InP around telecom wavelengths, a value of $n_{T}=2.01 \times 10^{-4} \mathrm{~K}^{-1}$ has been reported at room temperature.[49]

The combination of all these nonlinear processes is dominated by carrier-induced effects, namely plasma dispersion and band-filling processes due to the carriers generated by TPA, resulting in a reduction of the refractive index of the cavity. This leads to a blue shift of the cavity resonance frequency. An experimentally observed resonance shift of $\sim 1 \mathrm{~nm}$ [50] corresponds to changes in refractive index of around $\Delta n=n \Delta \lambda / \lambda_{0} \approx 2 \times 10^{-3}$, where $\lambda_{0}=$ $1550 \mathrm{~nm}$, and $n=3.17$ for InP.[46] In the next section, we discuss how these free-carriers relax, governing the recovery of the switch to its initial transmission spectrum.

\subsection{Free-carrier dynamics}

The initial free-carriers distribution in the nanocavity, generated by TPA, shows spatial and temporal variations due to carrier diffusion and recombination processes. In the absence of doping and an applied external electric field, carriers are assumed to spread and distribute homogenously within the membrane.[45] Additionally, within the ambipolar approximation electrons and holes are assumed to have the same distribution, i.e. $N(r, t)=P(r, t)$, where $N(r, t)$ is the distribution of electrons, and $P(r, t)$ is the distribution of holes.

The time evolution of free-carriers can be modelled by using a standard drift-diffusion rate equation model [51] as

$$
\dot{N}(r, t)=G(t)-D_{a} \nabla^{2} N(r, t)-N(r, t) / \tau_{e f f},
$$

where $D_{a}=\left(D_{n} \mu_{p}+D_{p} \mu_{n}\right) /\left(\mu_{p}+\mu_{n}\right)$ is the ambipolar diffusion coefficient, with $D_{n, p}$ and $\mu_{n, p}$ being the diffusion coefficient and mobility of electrons and holes, respectively. The carrier generation term $G(t)$ is related to the TPA coefficient and can be written using Equation 7 as

$$
G(t)=\frac{\beta_{T P A} I^{2}}{2 \hbar \omega}
$$

where $I$ is related to the energy stored in the cavity $|a(t)|^{2}$ as $I=c_{0}|a(t)|^{2} / n V_{T P A}$, where $c_{0}$ is the speed of light in vacuum, and $V_{T P A}$ is the effective TPA mode volume characterizing the free-carrier density distribution generated by TPA.[33] At the surface of the photonic crystal 


\section{WILEY-VCH}

membrane, Neumann boundary conditions are applied, i.e. $D_{a} \nabla N(r, t)=-S N(r, t)$, where $S$ is the surface recombination velocity.[33] Figure 7(a) shows the time-evolution of the carrier distribution around the $\mathrm{H} 0$ cavity at 0 ps, 5 ps and 50 ps. The initial distribution of free carriers is determined by the spatial profile of the excited cavity mode and is calculated using the $2 \mathrm{D}$ finite element method.[33] For this simulation, $S=0$, and $\tau_{\text {eff }} \rightarrow \infty$, so that the carrier distribution appears as a result of diffusion.[33]

(a)

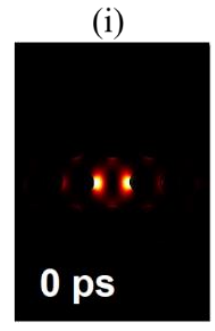

(b)

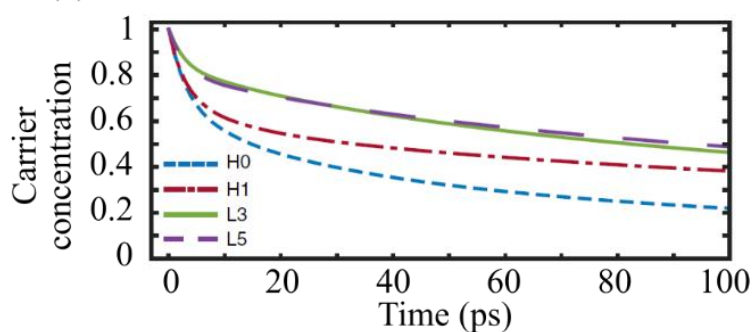

(ii)

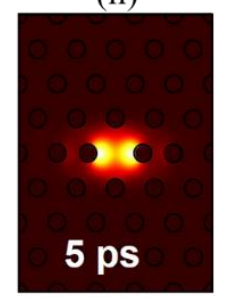

(c)

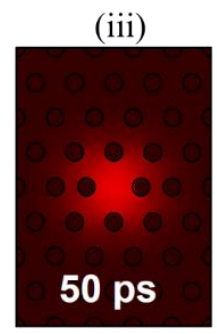

(i) $\mathrm{H} 0$

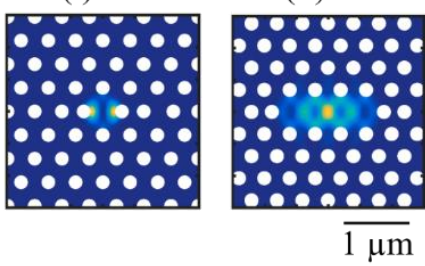

Figure 7: (a) Carrier density distribution around the H0 photonic crystal cavity at (i) 0 ps, (ii) 5 ps, and (iii) $50 \mathrm{ps}$ as determined by diffusion. (b) The decay of the carrier concentration for various InP based PhC cavities with $S=10 \mathrm{~m} / \mathrm{s}$. (c) Initial distribution of free-carriers for (i) H0 cavity, and (ii) L3 cavity. Higher carrier density is indicated by the bright yellow colour. (a) Reproduced with permission [33], Copyright 2013, The Optical Society. (b) and (c) Reproduced with permission [45], Copyright 2006, The American Physical Society.

The recombination or relaxation of carriers, the last term in Equation 9, includes contributions from bulk and surface recombination. Bulk recombination, due to radiative and Auger recombination, is a slow process with characteristic time scales spanning from tens to several hundreds of nanoseconds.[33], [52] An important carrier relaxation process in $\mathrm{PhC}$ structures, owing to their large surface area to volume ratio, is the surface recombination.[36] At the surface of a semiconductor the atomic lattice is abruptly interrupted. This results in dangling bonds, i.e. unpaired outer-shell electrons, which introduce surface states that can trap charge carriers enhancing electron-hole recombination.[53] The effective carrier lifetime, $\tau_{e f f}$, can then be written as [36] 


\section{WILEY-VCH}

$$
\frac{1}{\tau_{e f f}}=\frac{1}{\tau_{r e c}}+\frac{S}{L}
$$

where $\tau_{\text {rec }}$ is the bulk recombination rate, $S$ is the surface recombination velocity, and $L$ is the maximum distance to the top or bottom surface of the photonic crystal. For PhC membrane structures, $L=h / 2$, where $h$ is the PhC membrane thickness. Depending on the material and fabrication process, surface recombination may be more important than bulk recombination. GaAs, in particular, has a large surface recombination velocity of around $10^{7} \mathrm{~cm} \mathrm{~s}^{-1}$, and the carrier relaxation process is dominated by surface recombination, resulting in shorter carrier recovery time.[45] However, if the recovery time is significantly shorter than the modulating signal, the build-up of carrier density in the cavity region is reduced, which lowers the refractive index changes and diminishes the effect of the modulation. Due to this, GaAs based $\mathrm{PhC}$ nanocavities need to be coated with a few nanometers thick layer of alumina using atomic layer deposition (ALD) in order to achieve larger modulation.[36] The situation is different for InP with much lower surface recombination velocity of around $10^{3} \mathrm{~cm} \mathrm{~s}^{-1}$.[45] In this case, larger accumulation of carriers and hence larger modulation can be achieved at the expense of slower carrier recovery.

The impact of cavity size on the free-carrier dynamics of InP PhC cavities is illustrated in Figure 7(b) and Figure 7(c) taken from Ref.[45] It can be seen that the effective density of carriers decays much faster for smaller cavities compared to larger cavities.[45] The initial distributions of free carriers are shown in Figure 7(c) for H0 and L3 cavities. For the case of the HO cavity, carriers are generated close to the airholes, where they can recombine, while for the L3 cavity carriers have to diffuse longer before they exit the cavity or reach recombination sites.[45] Therefore, the cavity size plays an important role in realizing a fast optical switch. Detailed descriptions of the free-carrier dynamics in PhC cavities can be found in Ref. [31], [33], [36], [45], [54]

\section{All-optical signal processing using Fano devices}

In this section, we present some of the recent all-optical signal processing applications of Fano resonances.

\subsection{Pulse carving}




\section{WILEY-VCH}

Short pulses are often desired in optical communication systems for generating return-to-zero (RZ) signals, which have several advantages over non-return-to-zero (NRZ) signals in terms of improved receiver sensitivity, less inter-symbol interference, and lower average operating power.[55], [56] Short pulses can also be used for synchronization and clocking applications which require sharp raising and falling pulse edges or for obtaining precise information about the time of arrival of a signal.[56] In a conventional pulse carving scheme, the NRZ input signal is carved into a RZ signal using a radio frequency (RF) signal for modulation.[55], [57] Here, we discuss the use of a Fano resonance for carving of a short pulse from a long input pulse, without the need for an additional control signal.[50]

A red-parity Fano resonance structure is used to carve a long duration input pulse spectrally centered around the peak transmission of the Fano resonance. Figure 8(a) shows the measured output pulses as the power of the input pulse is gradually increased. When the power coupled to the cavity from the leading edge of the input pulse becomes large enough, the intracavity field efficiently generates a large number of free-carriers, leading to a blueshift of the resonance spectrum. This results in suppressed transmission for the remainder part of the pulse, leading to a short pulse. Using this scheme, short pulses of $\approx 20$ ps and 30 ps duration have been carved out of 93 ps and 500 ps long input pulses, respectively.[50]

\subsection{Signal reshaping}

Signal reshaping is a process by which the quality of a degraded data signal can be improved by noise suppression and extinction ratio improvement [58]. Efficient signal reshaping can be performed electrically by detecting the noisy optical signal and retransmitting it back in the optical domain.[59], [60] However, this process is energy-consuming due to the use of optoelectronic conversion units, in addition to limiting the signal transmission rate.[59], [61] On the other hand, an all-optical implementation of signal reshaping offers substantial benefits due to potentially faster processing speed, while eliminating optical-to-electrical conversion.[59], [61], [62] Here, we discuss the use of a Fano resonance for suppressing amplitude fluctuations of the ' 1 ' bit of optical data signals.

A red-parity Fano resonance exhibits a nonlinear power transfer function as a result of the combination of the asymmetric Fano lineshape and the carrier-induced nonlinear effects in the nanocavity [41]. Using this nonlinear power transfer function, suppression of amplitude 


\section{WILEY-VCH}

fluctuations of RZ data signals at $2 \mathrm{Gbit} / \mathrm{s}$ and $10 \mathrm{Gbit} / \mathrm{s}$ have been demonstrated.[41] The results of the signal reshaping experiment are shown by the eye diagrams of Figure 8(b). It shows the reference signal (top), degraded signal (middle), and reshaped signal (bottom). Note that the amplitude fluctuations of the ' 1 ' bit level are reduced, improving the eye opening of the reshaped signal compared to the degraded signal.

(a) Pulse carving

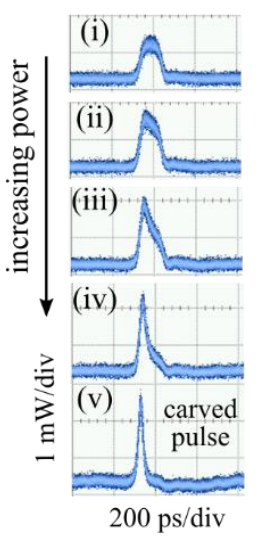

(b) Signal reshaping

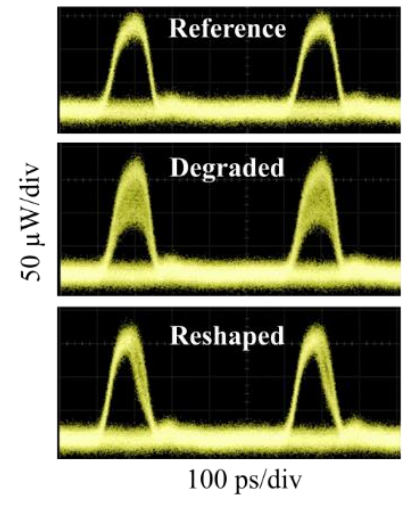

(c) Wavelength conversion

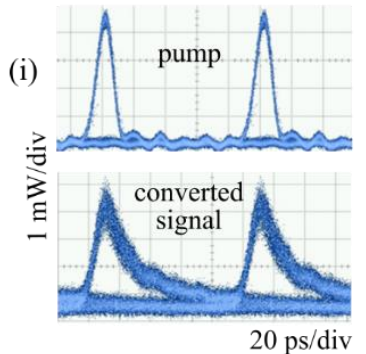

(ii)

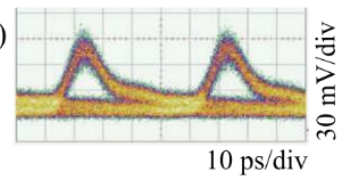

(d) OTDM demultiplexing
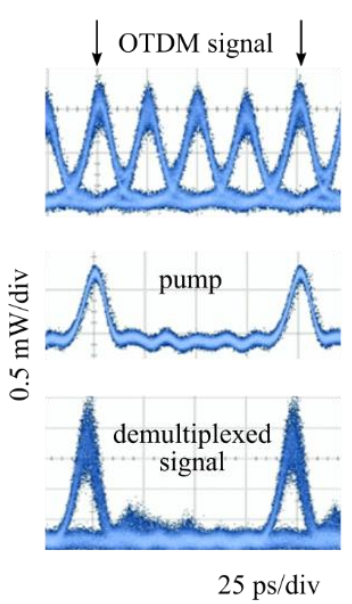

Figure 8: All-optical signal processing using Fano devices. (a) Self-pulse carving. [50] (b) Signal reshaping of $2 \mathrm{Gbit} / \mathrm{s}$ data signals. (c) $10 \mathrm{Gbit} / \mathrm{s}$ and $20 \mathrm{Gbit} / \mathrm{s}$ wavelength conversion. (d) $40 \mathrm{Gbit} / \mathrm{s}$ to 10 Gbit/s optical time domain demultiplexing. (b) Reproduced with permission [41], Copyright 2018, The Optical Society. (c) Part (i) Reproduced with permission [63], Copyright 2017, SPIE. Part (ii) Reproduced with permission [43], Copyright 2015, The Optical Society. (d) Reproduced with permission [64], Copyright 2017, IEEE.

\subsection{Wavelength conversion}

Wavelength conversion involves the transfer of an optical data signal (bit pattern) from one wavelength to another, without compromising the data.[60] In this subsection, we use the principles of wavelength conversion to illustrate the application of a Fano resonance as an alloptical switch.

A modulated data signal, acting as a pump, is spectrally located at the transmission peak of a blue parity Fano resonance, while a continuous wave (CW) probe signal is located at the transmission minimum, as illustrated in Figure 9(a). Here, the spectral location of the pump is chosen to match the resonance frequency of the cavity, which can be extracted from the CMT 


\section{WILEY-VCH}

fit to the measured transmission spectrum. This allows efficient coupling of the pump signal to the cavity resulting in strong carrier-induced nonlinear processes. When the pump signal represents a ' 0 ' bit, the resonance is unshifted and a CW probe signal spectrally located at the minimum transmission of the Fano resonance will show suppressed transmission, representing the switch-off state of the Fano switch. However, when the pump signal represents a logical bit ' 1 ', it will induce a resonance blue-shift. This results in maximum transmission for the $\mathrm{CW}$ probe signal corresponding to the switch-on case. Figure 9(b) shows the normalized spatial distribution of the $E_{y}$ field component, calculated using the 3D finite difference time domain (FDTD) method at the Fano transmission peak, panel (i), and the transmission minimum, panel (ii). For better illustration, the photonic crystal device is overlapped with the field distribution. The $E_{y}$ field distribution at the Fano peak shows high transmission to the output port, while the $E_{y}$ field distribution at the Fano minimum indicates low transmission to the output port, since most of the power is reflected back to the input port due to destructive interference.

The eye diagrams of the pump and the wavelength-converted $10 \mathrm{Gbit} / \mathrm{s}$ signals are shown in Figure 8(c) panel (i). Error-free $10 \mathrm{Gbit} / \mathrm{s}$ wavelength conversion with energy consumption down to $72 \mathrm{fJ} / \mathrm{bit}$ [63] and $60 \mathrm{fJ} / \mathrm{bit}$ [43] have been demonstrated. Similarly, $20 \mathrm{Gbit} / \mathrm{s}$ wavelength conversion with bit-error ratio of $5 \times 10^{-4}$ well below the hard-decision forward error correction limit has been demonstrated with energy consumption down to $134 \mathrm{fJ} / \mathrm{bit}$ [43]. The eye diagram of the $20 \mathrm{Gbit} / \mathrm{s}$ wavelength converted signal is shown in Figure 8(c) panel (ii). It should be noted that these wavelength conversion experiments are used as proof of concept demonstrations for all-optical switching using Fano resonance. However, the use of Fano resonance as wavelength converter is limited due to smaller conversion bandwidth, i.e. the Fano peak to minimum spectral separation. A more practical application of a Fano resonance is its use for optical time domain demultiplexing, as discussed in the following subsection. 


\section{WILEY-VCH}

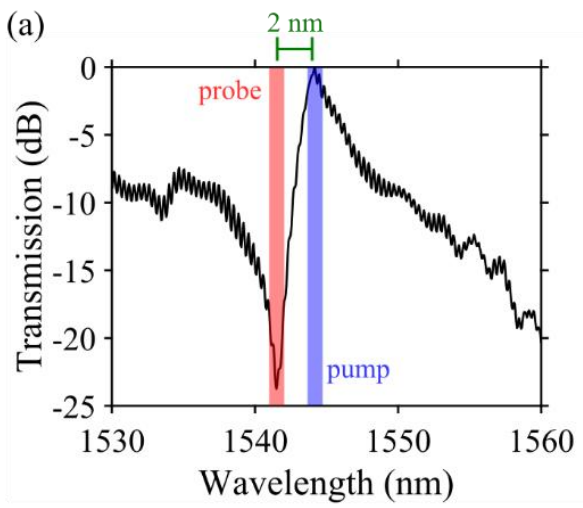

(b)

(i)

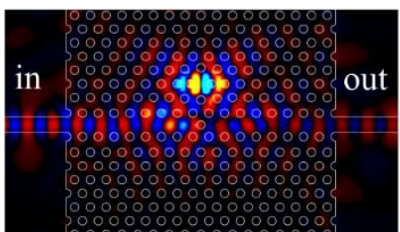

(ii)

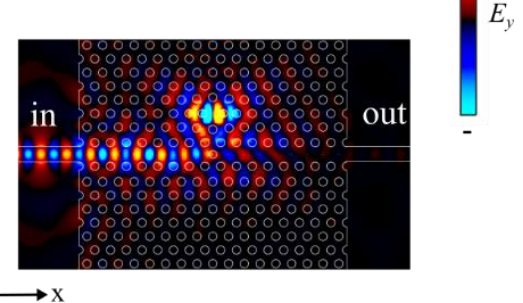

Figure 9: Asymmetric Fano resonance and its $E_{y}$-field distributions. (a) Measured transmission in dB, showing an extinction ratio of around $24 \mathrm{~dB}$. The wavelength separation between the peak and the minimum transmission point is around $2 \mathrm{~nm}$. (b) Normalized $E_{y}$-field distribution calculated using 3D FDTD at Fano transmission peak (i), and transmission minimum (ii).

\subsection{Optical time domain demultiplexing}

Optical time domain multiplexing (OTDM) is a technique in which $N$ optical signals at the same bit rate $B$ and sharing the same carrier frequency are multiplexed to form a composite stream of bits at the bit rate of $N \times B$.[60] This allows high speed optical transmission and efficient use of the available transmission bandwidth.[65] At the receiver end, each of the OTDM channels need to be detected without interference from the neighboring channels. Due to limited electronic receiver bandwidth, it is difficult to detect a single channel directly from a very high speed OTDM signal.[66] This sets a limit to the highest bit rate at which the system can be operated. Alternatively, optical time domain demultiplexing can be used to separate the high speed OTDM signal into individual channels, which can then easily be detected by the electronic receiver.[65], [67] In this subsection, we discuss the application of a Fano switch for optical time domain demultiplexing.

Using a blue-parity Fano resonance, a $40 \mathrm{Gbit} / \mathrm{s}$ OTDM signal is placed spectrally at the minimum of the Fano resonance, and a train of $10 \mathrm{GHz}$ pulse acting as pump signal is spectrally located around the peak of the Fano resonance. An optical delay line is used to overlap the pump signal with one of the OTDM channels to be demultiplexed. The eye diagrams of the $40 \mathrm{Gbit} / \mathrm{s}$ OTDM (top), $10 \mathrm{GHz}$ pump (middle) and the demultiplexed 10 Gbit/s (bottom) signals are shown in Figure 8(d). When a pump pulse excites the cavity, a 


\section{WILEY-VCH}

blue-shift of the Fano resonance occurs. This allows a particular OTDM channel to be transmitted. As soon as the pump pulse leaves the cavity, the resonance shifts back, blocking the transmission for the remaining channels. Using this scheme, error-free performance with switching energy as low as $63 \mathrm{fJ} / \mathrm{bit}$ has been demonstrated.[64] While the optical time domain demultiplexing from $40 \mathrm{Gbit} / \mathrm{s}$ to $10 \mathrm{Gbit} / \mathrm{s}$ using a Fano resonance serves as a proofof-principle demonstration, demultiplexing at data rates $>80 \mathrm{Gbit} / \mathrm{s}$ is required for practical applications. This is because current electronic receiver technology can detect a $40 \mathrm{Gbit} / \mathrm{s}$ signal without the need for optical demultiplexing.[65], [66] In Section 6, we discuss possible ways to improve the switching speed of the devices.

A comparison of all-optical switching demonstrations on various nonlinear platforms is presented in Table 1. The comparison criteria include resonance lineshape, nonlinear process, demonstrated application, operation speed, and switching energy. The experimental demonstrations can broadly be classified as the study of device dynamics using pump-probe techniques, and all-optical signal processing demonstrations. The pump-probe technique allows for characterizing switch recovery times and estimation of switching energy, and by using low repetition rates it can be considered as single-pulse experiment. On the other hand, all-optical signal processing experiments truly challenge the device performance by using GHz repetition rates. From these demonstrations, Fano devices reported in [43], [64] show the highest operation speed with low switching energy required for error-free performance. These results indicate that Fano devices are indeed potential candidates for future all-optical switching. Note that, the smallest switching energy reported so far is $20.4 \mathrm{fJ}$ using a Lorentzian resonance.[6] However, this demonstration does not include bit error ratio measurements, making it difficult to compare the reported switching energy to similar Fano devices. 


\section{WILEY-VCH}

Table 1: Comparison of all-optical switches realized using Fano and Lorentzian resonances.

\begin{tabular}{|c|c|c|c|c|c|c|}
\hline Platform & $\begin{array}{l}\text { Resonance } \\
\text { lineshape }\end{array}$ & $\begin{array}{l}\text { Nonlinear } \\
\text { process }\end{array}$ & Application & $\begin{array}{l}\text { Operation } \\
\text { speed }\end{array}$ & $\begin{array}{l}\text { Switching } \\
\text { energy }\end{array}$ & Ref. \\
\hline $\begin{array}{l}\text { InP/SOI PhC } \\
+\mathrm{QW}\end{array}$ & Lorentzian & $\begin{array}{l}\text { Carrier- } \\
\text { induced }\end{array}$ & $\begin{array}{l}\text { Wavelength } \\
\text { conversion }\end{array}$ & $20 \mathrm{Gbit} / \mathrm{s}$ & - & [68] \\
\hline GaAs PhC & Lorentzian & $\begin{array}{l}\text { Carrier- } \\
\text { induced }\end{array}$ & Pump-probe & $5 \mathrm{GHz}$ & $190 \mathrm{fJ}$ & [36] \\
\hline InP PhC & Fano & $\begin{array}{l}\text { Carrier- } \\
\text { induced }\end{array}$ & $\begin{array}{l}\text { Wavelength } \\
\text { conversion }\end{array}$ & $10(20) \mathrm{Gbit} / \mathrm{s}$ & $\begin{array}{l}60 \quad(134 *) \\
\text { fJ/bit }\end{array}$ & [43] \\
\hline GaInP PhC & - & $\begin{array}{l}\text { Kerr effects } \\
(\mathrm{FWM})\end{array}$ & demultiplexing & $\begin{array}{l}10.56 \mathrm{Gbit} / \mathrm{s} \text { to } \\
330 \mathrm{Mbit} / \mathrm{s}\end{array}$ & - & [69] \\
\hline $\begin{array}{l}\text { InGaAsP } \\
\mathrm{PhC}\end{array}$ & Lorentzian & $\begin{array}{l}\text { Carrier- } \\
\text { induced }\end{array}$ & $\begin{array}{l}\text { Pulse extraction } \\
\text { and rejection }\end{array}$ & $\begin{array}{l}40 \mathrm{GHz} \text { to } 10 \\
\mathrm{GHz}\end{array}$ & $20.4 \mathrm{fJ}$ & {$[6]$} \\
\hline $\begin{array}{l}\mathrm{Si} \quad \mathrm{PhC} \\
\text { nanobeam }\end{array}$ & Lorentzian & $\begin{array}{l}\text { Carrier- } \\
\text { induced }\end{array}$ & $\begin{array}{l}\text { Pulse extraction } \\
\text { and rejection }\end{array}$ & $\begin{array}{l}2.5 \mathrm{GHz} \text { to } \\
100 \mathrm{MHz}\end{array}$ & $1.6 \mathrm{pJ}$ & [37] \\
\hline $\mathrm{Si} \mathrm{PhC}$ & Fano & $\begin{array}{l}\text { Carrier } \\
\text { induced }\end{array}$ & $\begin{array}{l}\text { Pulse extraction } \\
\text { and rejection }\end{array}$ & $2.5 \mathrm{GHz}$ & $80 \mathrm{fJ}$ & [30] \\
\hline GaAs PhC & Lorentzian & $\begin{array}{l}\text { Carrier- } \\
\text { induced }\end{array}$ & Pump-probe & - & $120 \mathrm{fJ}$ & [32] \\
\hline InP PhC & Fano & $\begin{array}{l}\text { Carrier- } \\
\text { induced }\end{array}$ & demultiplexing & $\begin{array}{l}40 \mathrm{Gbit} / \mathrm{s} \text { to } 10 \\
\mathrm{Gbit} / \mathrm{s}\end{array}$ & $63 \mathrm{fJ} / \mathrm{bit}$ & [64] \\
\hline $\begin{array}{l}\mathrm{a}-\mathrm{Si}: \mathrm{H} \\
\text { microring }\end{array}$ & Lorentzian & $\begin{array}{l}\text { Kerr effects } \\
(\mathrm{XPM})\end{array}$ & Pump-probe & - & $720 \mathrm{fJ}$ & {$[70]$} \\
\hline $\begin{array}{l}\text { Si-rich } \mathrm{SiNx} \\
\text { microring }\end{array}$ & Lorentzian & Kerr effects & $\begin{array}{l}\text { Wavelength } \\
\text { conversion }\end{array}$ & $12 \mathrm{Gbit} / \mathrm{s}$ & - & [71] \\
\hline
\end{tabular}

* indicates switching energy measured under non-error free conditions

\section{Fano diodes - non-reciprocal transmission properties}

In the effort towards building complex photonic integrated circuits, devices that feature nonreciprocal light transmission, i.e., isolation, are key building blocks to allow sectioning of the circuit. This is analogous to what is possible in electronic integrated circuits, in which each field-effect transistor provides isolation between input and output signals. Diverse 


\section{WILEY-VCH}

physical mechanisms and configurations have been proposed in order to break the reciprocity of linear optical devices and realize asymmetrical light propagation.[72]-[76] However, these structures typically suffer from high complexity, costly fabrication, high energy consumption, small bandwidth or large footprint.

Photonic crystal Fano structures with broken symmetry such as the one shown in Figure 5(c), on the other hand, offer a simple and compact solution.[77] Here, the direction selectivity of the Fano structure is exploited, i.e., light transmission from left to right, forward transmission, differs substantially from the transmission from right to left, backward transmission, cf. Figure 10(a). The mirror symmetry of the structure is broken by displacing the PTE by one lattice constant towards the left. This makes the coupling rate between port 1 and the cavity larger than between port 2 and the cavity.[77] As a result, the magnitude of the carrier-induced resonance shift is different for the two propagation directions leading to nonreciprocal transmission. The basis for the transmission asymmetry is therefore the combination of spatial asymmetry and nonlinearity.

Figure 10(b) shows the input-output power transfer function for forward (green line) and backward (red line) transmission of a CW signal spectrally located around the minimum of a blue parity Fano resonance. For both cases, the output power initially increases linearly with the input power. Upon increasing the input power, both the forward and backward transmissions show a step-like increase in transmission due to the blue shift of the resonance which results in the overlap of the input signal spectrum with the Fano transmission peak. However, this effect occurs at much lower input power levels for the forward transmission compared to the backward transmission. The shaded regions in Figure 10(b) indicate bistable transmission regions, for both the forward and backward transmissions, determined by measuring the output power for both increasing and decreasing input powers, indicated by the arrows. It can be noted that there is a range of input power levels between the two bistable regions, where the backward transmission is suppressed by around $30 \mathrm{~dB}$ compared to the forward transmission. 

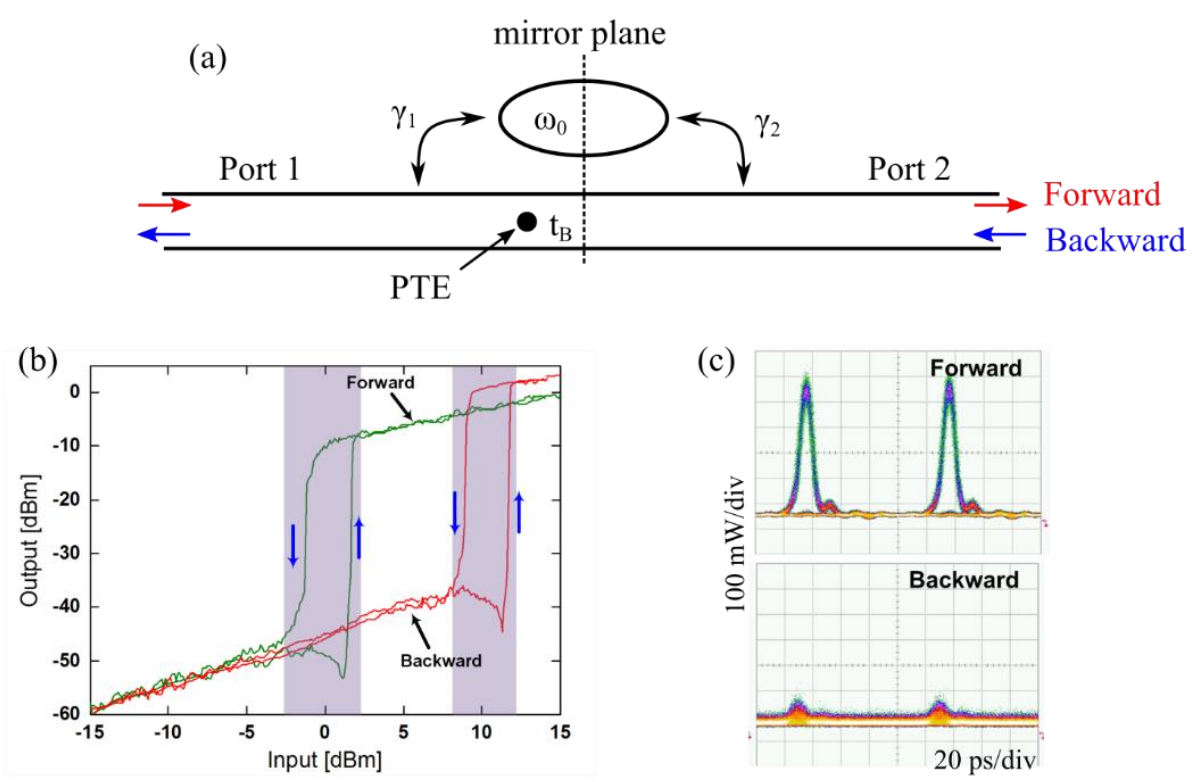

Figure 10: Fano diode structure and characteristics. (a) Schematic of the system with broken mirror symmetry achieved by displacing the PTE towards port 1. (b) non-reciprocal transmission under CW illumination. Measured output power versus input power for forward (green) and backward (red) direction. The shaded areas indicate the bistable regions. The non-reciprocal transmission region is located between the two bistable regions, and exhibits more than $30 \mathrm{~dB}$ non-reciprocal transmission ratio (NTR). (c) eye diagrams showing forward and backward transmission of a $10 \mathrm{Gbit} / \mathrm{s}$ modulated input data signal. (b) Reproduced with permission [63], Copyright 2017, SPIE. (c) Reproduced with permission [77], Copyright 2015, Wiley-VCH.

The non-reciprocal transmission effect is also investigated using dynamic characterization in which a $10 \mathrm{Gbit} / \mathrm{s} \mathrm{RZ}$ signal is injected into the device in the forward and backward directions. The eye diagrams in Figure 10(b) show the signal transmission in the forward (top), and backward (bottom) direction. A clear and open eye diagram is observed in the forward direction, while the backward transmission is low with completely closed eye diagram. A non-reciprocal transmission ratio (NTR) of approximately $10 \mathrm{~dB}$ is achieved with input energy as low as $4.5 \mathrm{fJ} / \mathrm{bit} .[77]$

It should be noted that although a large NTR can be achieved, such structure is actually reciprocal when two CW signals are injected from both sides simultaneously.[77] For pulsed signals, by exploiting ultrafast carrier effects with a typical relaxation time on the order of tens to hundred picoseconds, the device can suppress backward propagating pulses in the time interval between two neighbouring forward pulses. This may be used to isolate pulsed sources, like mode-locked lasers. Nevertheless, it still remains a challenge to realize nonreciprocal 


\section{WILEY-VCH}

transmission during the time slot of the forward propagating pulse using structures relying on nonlinearities.

\section{Fano lasers}

Another novel application of Fano resonances is the realization of ultra-small lasers featuring a narrowband mirror based on Fano interference between a continuum of waveguide modes and the discrete resonance of a nanocavity.[78], [79] This leads to unique laser characteristics such as single mode operation, and generation of a self-sustained train of pulses at gigahertz frequencies. SEM image of a fabricated Fano laser is shown in Figure 11(a). It is a $250 \mathrm{~nm}$ thick InP photonic crystal membrane which consists of a line defect cavity, and three layers of InAs quantum dots as gain material. The left mirror of the cavity is a broadband mirror realized by blocking the waveguide with airholes, similar to a conventional line-defect photonic crystal laser. The right mirror is realized by Fano interference between a discrete mode in the nanocavity and a continuum of waveguide modes. Due to destructive interference between the light transmitted through the waveguide and the nanocavity, a narrowband reflection centered around the resonance frequency of the nanocavity is created.[79] This narrowband reflection results in single mode operation of the laser.

The sample is optically pumped using CW light from the top using an objective lens. The pumping area is indicated by the dashed yellow circle in Figure 11(a). The static characteristic of the Fano laser is shown in Figure 11(b), where the measured output peak power versus pump power is indicated by red dots, while the black line indicates a theoretical fit using the conventional rate equation model, from which the spontaneous emission factor is estimated to be around $1 \%$.[78] A clear transition to lasing is observed at a threshold pump power of around $4.6 \mathrm{dBm}$. Figure 11(c) shows measured spectra for pump powers of $3 \mathrm{dBm}$ (blue) and $7.5 \mathrm{dBm}$ (red) showing operation below and above the lasing threshold, respectively. A single dominant mode is observed at $1566 \mathrm{~nm}$.

Furthermore, the laser displays rich dynamics upon modulation of the nanocavity resonance. It is theoretically predicted that for small-amplitude modulation of the nanocavity resonance, the laser can be operated at $\mathrm{THz}$ frequencies.[79] For larger modulation amplitudes, short pulse generation is anticipated.[79] Detailed theoretical investigations of the Fano laser are provided in Ref.[80]-[83] 


\section{WILEY-VCH}

(a)

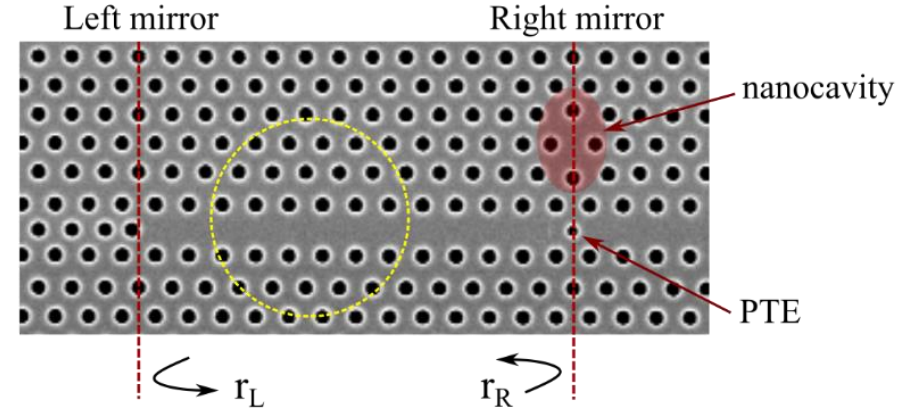

(b)

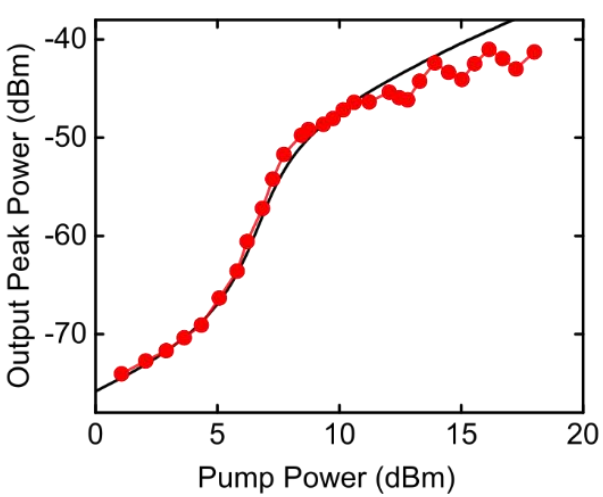

(c)

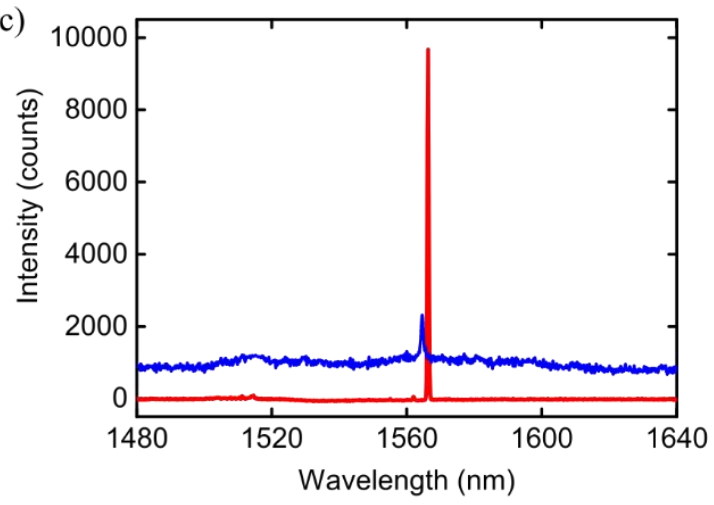

Figure 11: InP photonic crystal Fano laser. (a) SEM image of the fabricated Fano laser consisting of a line-defect waveguide terminated by a broadband left mirror and a Fano mirror on the right. The Fano mirror is composed of a nanocavity side-coupled to the line-defect waveguide forming a narrowband mirror. (b) Measured output peak power versus pump power. Red dots indicate experimental data while black solid line indicates theoretical fit using a conventional rate equation model. (c) Measured spectra for pump powers of $3 \mathrm{dBm}$ (blue) and $7.5 \mathrm{dBm}$ (red) showing operation below and above the lasing threshold, respectively. For clarity the blue curve is shifted vertically. (a) - (c) Reproduced with permission [78], Copyright 2016, Springer Nature.

\section{Discussion and future perspectives}

Resonance-based all-optical switches are good candidates for future chip-to-chip or on-chip optical communication networks in which low energy consumption and high-speed performance are desired. However, significant research effort is still required in order to reach devices of practical value. In this section, we discuss approaches to improve the switching speed of free-carrier based devices, new device functionalities envisaged using Fano structures, and a guideline for reporting switching energies of all-optical signal processing devices.

As discussed in Section 2, the accumulated free-carriers relax with time scales determined by the carrier recombination and diffusion rates. This sets a limit to the highest 


\section{WILEY-VCH}

switching speed that can be achieved as a complete recovery of the resonance shift should occur before the next pulse arrives at the nanocavity. As surface recombination is the dominant free-carrier relaxation process, it is advantageous to increase the surface area to volume ratio by implementing the devices on thinner photonic crystal membrane, such as 250 $\mathrm{nm}$ rather than $340 \mathrm{~nm}$ thick membranes. One of the approaches to address the slow recovery of the switch is the transition from the ambipolar diffusion regime to the faster minority carrier dominated diffusion regime. It is reported that a twofold acceleration of the carrier dynamics can be achieved using p-doping of the InP photonic crystal.[84] Another possibility is to sweep carriers out of the cavity region by applying a static electric field across the nanocavity. This can be implemented using either a p-i-n junction or a metal-semiconductormetal (MSM) structure. In fact, such implementations are very well known for carrier extraction across standard strip waveguides [85] and photonic crystal cavities.[86], [87] A combination of these technologies with Fano switches could potentially help in achieving faster all-optical switches.

Alternatively, the slow switching tails can be suppressed by applying blue-detuned spectral filtering on the output signal.[37] This can be understood by considering the switching process, in which a pump pulse injected into the nanocavity generates free-carriers at much higher rate than the carrier relaxation process. These fast and slow-changing phase shifts lead to large blue-chirped and a small red-chirped components of the probe signal, respectively, via transient cross-phase modulation.[37], [88] By filtering out the small redchirped component associated with the slow recovery of the switch using an optical band pass filter, improvement on the switching performance of Lorentzian based all-optical switches is demonstrated in Ref.[37] Implementation of such blue-spectral filtering on Fano devices could potentially improve their switching performances without having to change the device platform. However, it does not speed up the response time of the switch which is intrinsic to the device platform.

A wide range of new device functionalities can be realized by exploiting the sharp asymmetric lineshape of Fano resonances. Of particular interest are electro-optic modulators using Fano resonances. By injecting carriers into the cavity region via p-i-n structures, electrically controlled Fano modulators can be realized. The expected key advantages include low power consumption at $\mathrm{GHz}$ operation speeds, enabled by the use of small p-i-n structures 


\section{WILEY-VCH}

and high-Q nanocavities. However, technological challenges in fabricating such devices with high reproducibility remain important issues for further study, as do thermal effects, which introduce long time constants that may also limit the switching speed. Implementations of carrier injection based electro-optic modulators using Lorentzian resonances have already been reported with operating power in the $\mu \mathrm{W}$ level.[89] Furthermore, the same device structure is used to realize high quantum efficiency photodetectors.[90] Similar implementations of electro-optic Fano modulators are envisaged.

The design of high bitrate optical modulators using resonant structures is challenging due to the trade-off between the optical bandwidth of the nanocavity and the achievable modulation bandwidth. High Q-factor nanocavities enable low power operation, but the resulting long photon lifetime is detrimental to high speed operation.[25] Similar trade-off exists for optical switches such that the spectral bandwidth of the data signal should be smaller than the linewidth of the resonance in order to achieve complete suppression and transmission for switch-off and switch-on operations, respectively. Nevertheless, it is envisaged that the implementation of efficient carrier injection and sweep-out technologies with Fano resonance devices should enable operations up to $100 \mathrm{Gbit} / \mathrm{s}$ with Q-factors of only a few thousands, corresponding to resonance linewidth in the range of 1-2 nm.

When comparing devices of various structures, it is common to evaluate the energy required for the switching action of the device. Particularly for optical signal processing applications, error counts or bit-error ratio (BER) measurements are essential to quantify the performance of the devices. Some of these performance metrics include the switching energy required to operate the device for error-free performance, the $1 / e$ recovery time of the switch, and the power penalty incurred as a result of using the device compared to a reference measurement. Therefore, we recommend that switching energy reports shall also include the BER value at which the all-optical switching is performed.

\section{Summary}

This review discusses a class of photonic crystal devices employing Fano resonances. Particular focus is given to in-plane device concepts as relevant for future integrated photonic chips. Different types of Fano resonance lineshapes are introduced and classified, and the general theory as well as recent experimental demonstrations are discussed. Unlike other Fano 


\section{WILEY-VCH}

structures which require topological protection to ensure ultrasharp asymmetric lineshapes [91], the photonic crystal Fano structures are robust to fabrication imperfections and ensure the existence of Fano resonances. Due to the similar device physics and geometrical similarity with Lorentzian resonance structures, the review also discusses various implementations of Lorentzian resonances, highlighting potential advantages of Fano devices over Lorentzian devices for all-optical switching applications. The nonlinear and dynamical characteristics of the nanocavities featuring free-carrier generation via TPA, carrier-induced refractive index changes, and the associated carrier relaxation processes are discussed. The unique features of Fano lineshapes in combination with nanocavity-enhanced optical nonlinear processes have brought about a wide range of all-optical signal processing applications. In most of these applications, devices based on Fano resonances have shown improved performance compared to conventional devices based on a Lorentzian resonance. Furthermore, different avenues for further improving the switching performances of free-carrier based optical switches have been identified, and future perspectives of the devices are discussed.

\section{Acknowledgements}

The authors would like to thank Hao Hu, Michael Galili, and Leif Katsuo Oxenløwe for assistance with system experiments. The authors acknowledge financial support from Villum Fonden via the NATEC (Nanophotonics for Terabit Communications) centre under grant number 8692. This project has received funding from the European Research Council (ERC) under the European Union's Horizon 2020 research and innovation programme (grant agreement number 834410 - FANO).

Keywords: Fano resonances, photonic crystals, all-optical switching, carrier-induced nonlinearities, free-carrier dynamics

\section{References}

[1] O. Painter, R. K. Lee, A. Scherer, A. Yariv, J. D. O'brien, P. D. Dapkus, and I. Kim, Science (80-. )., vol. 284, no. 5421, pp. 1819-1821, 1999.

[2] T. Baba, Nat. Photon., vol. 2, no. 8, pp. 465-473, Aug. 2008.

[3] B. E. Little, S. T. Chu, H. A. Haus, J. Foresi, and J.-. Laine, J. Light. Technol., vol. 15, 


\section{WILEY-VCH}

no. 6, pp. 998-1005, 1997.

[4] P. Michler, A. Kiraz, C. Becher, W. V Schoenfeld, P. M. Petroff, L. Zhang, E. Hu, and A. Imamoglu, Science (80-. )., vol. 290, no. 5500, pp. 2282-2285, 2000.

[5] H.-G. Park, S.-H. Kim, S.-H. Kwon, Y.-G. Ju, J.-K. Yang, J.-H. Baek, S.-B. Kim, and Y.-H. Lee, Science (80-. )., vol. 305, no. 5689, pp. 1444-1447, 2004.

[6] K. Nozaki, T. Tanabe, A. Shinya, S. Matsuo, T. Sato, H. Taniyama, and M. Notomi, Nat. Photon., vol. 4, no. 7, pp. 477-483, 2010.

[7] L. Novotny and B. Hecht, Principles of nano-optics, Cambridge University Press, 2012.

[8] A. E. Miroshnichenko, S. Flach, and Y. S. Kivshar, Rev. Mod. Phys., vol. 82, no. 3, pp. 2257-2298, 2010.

[9] Y. Akahane, T. Asano, B.-S. Song, and S. Noda, Nature, vol. 425, p. 944, Oct. 2003.

[10] J. S. Foresi, J. Villeneuve, P. R.; Ferrera, E. R. Thoen, G. Steinmeyer, S. Fan, J. D. Joannopoulos, L. C. Kimerling, H. I. Smith, and E. P. Ippen, Nature, vol. 390, no. 6656, pp. 143-145, 1997.

[11] P. Lalanne, C. Sauvan, and J. P. Hugonin, Laser Photon. Rev., vol. 2, no. 6, pp. 514$526,2008$.

[12] F. Wang, R. E. Christiansen, Y. Yu, J. Mørk, and O. Sigmund, Appl. Phys. Lett., vol. 113, no. 24, p. 241101, 2018.

[13] H. Choi, M. Heuck, and D. Englund, Phys. Rev. Lett., vol. 118, no. 22, p. 223605, 2017.

[14] S. Hu and S. M. Weiss, ACS photonics, vol. 3, no. 9, pp. 1647-1653, 2016.

[15] B. Luk'yanchuk, N. I. Zheludev, S. A. Maier, N. J. Halas, P. Nordlander, H. Giessen, and C. T. Chong, Nat. Mater., vol. 9, no. 9, pp. 707-715, 2010.

[16] C. Wu, A. B. Khanikaev, R. Adato, N. Arju, A. A. Yanik, H. Altug, and G. Shvets, Nat. Mater., vol. 11, no. 1, p. 69, 2012.

[17] J. W. Yoon and R. Magnusson, Opt. Express, vol. 21, no. 15, p. 17751, Jul. 2013.

[18] U. Fano, Phys. Rev., vol. 124, no. 6, pp. 1866-1878, 1961.

[19] M. F. Limonov, M. V. Rybin, A. N. Poddubny, and Y. S. Kivshar, Nat. Photon., vol. 11, no. 9, pp. 543-554, 2017.

[20] Jae Woong Yoon, Myoung Jin Jung, Seok Ho Song, and R. Magnusson, IEEE J. Quantum Electron., vol. 48, no. 7, pp. 852-861, Jul. 2012.

[21] A. D. Osterkryger, J. R. de Lasson, M. Heuck, Y. Yu, J. Mørk, and N. Gregersen, Opt. 


\section{WILEY-VCH}

Lett., vol. 41, no. 9, p. 2065, 2016.

[22] M. Galli, S. L. Portalupi, M. Belotti, L. C. Andreani, L. O’Faolain, and T. F. Krauss, Appl. Phys. Lett., vol. 94, no. 7, p. 71101, Feb. 2009.

[23] W. Zhou, D. Zhao, Y. C. Shuai, H. Yang, S. Chuwongin, A. Chadha, J. H. Seo, K. X. Wang, V. Liu, Z. Ma, and S. Fan, Prog. Quantum Electron., vol. 38, no. 1, pp. 1-74, 2014.

[24] D. A. B. Miller, J. Light. Technol., vol. 35, no. 3, pp. 346-396, 2017.

[25] J. Zhang, X. Leroux, E. Durán-Valdeiglesias, C. Alonso-Ramos, D. Marris-Morini, L. Vivien, S. He, and E. Cassan, ACS Photonics, vol. 5, no. 11, pp. 4229-4237, Nov. 2018.

[26] P. Yu, T. Hu, H. Qiu, F. Ge, H. Yu, X. Jiang, and J. Yang, Appl. Phys. Lett., vol. 103, no. 9, 2013.

[27] G. Dong, Y. Wang, and X. Zhang, Opt. Lett., vol. 43, no. 24, pp. 5977-5980, 2018.

[28] K. Nozaki, A. Shinya, S. Matsuo, T. Sato, E. Kuramochi, and M. Notomi, Opt. Express, vol. 21, no. 10, p. 11877, 2013.

[29] X. Yang, C. Husko, C. W. Wong, M. Yu, and D. L. Kwong, Appl. Phys. Lett., vol. 91, no. 5, pp. 1-4, 2007.

[30] K. Nozaki, E. Kuramochi, A. Shinya, and M. Notomi, Opt. Express, vol. 22, no. 12, pp. 14263-14274, 2014.

[31] A. De Rossi, M. Lauritano, S. Combrié, Q. V. Tran, and C. Husko, Phys. Rev. A - At. Mol. Opt. Phys., vol. 79, no. 4, pp. 1-9, 2009.

[32] C. Husko, A. De Rossi, S. Combrié, Q. V. Tran, F. Raineri, and C. W. Wong, Appl. Phys. Lett., vol. 94, no. 2, pp. 2007-2010, 2009.

[33] Y. Yu, E. Palushani, M. Heuck, N. Kuznetsova, P. T. Kristensen, S. Ek, D. Vukovic, C. Peucheret, L. K. Oxenløwe, S. Combrié, A. de Rossi, K. Yvind, and J. Mørk, Opt. Express, vol. 21, no. 25, pp. 31047-61, 2013.

[34] Y. Yu, E. Palushani, M. Heuck, D. Vukovic, C. Peucheret, K. Yvind, and J. Mork, Appl. Phys. Lett., vol. 105, no. 7, p. 71112, 2014.

[35] P. Colman, P. Lunnemann, Y. Yu, and J. Mørk, Phys. Rev. Lett., vol. 117, no. 23, p. $233901,2016$.

[36] G. Moille, S. Combrié, L. Morgenroth, G. Lehoucq, F. Neuilly, B. Hu, D. Decoster, and A. de Rossi, Laser \& Photon. Rev., vol. 10, no. 3, pp. 409-419, 2016. 


\section{WILEY-VCH}

[37] G. Dong, W. Deng, J. Hou, L. Chen, and X. Zhang, Opt. Express, vol. 26, no. 20, pp. 25630-25644, Oct. 2018.

[38] M. Heuck, P. T. Kristensen, Y. Elesin, and J. Mørk, Opt. Lett., vol. 38, no. 14, p. 2466, 2013.

[39] S. Fan, W. Suh, and J. D. Joannopoulos, JOSA A, vol. 20, no. 3, pp. 569-572, 2003.

[40] W. Suh, Z. Wang, and S. Fan, IEEE J. Quantum Electron., vol. 40, no. 10, pp. 1511$1518,2004$.

[41] D. A. Bekele, Y. Yu, H. Hu, P. Guan, M. Galili, L. Ottaviano, L. K. Oxenløwe, K. Yvind, and J. Mork, Opt. Express, vol. 26, no. 15, pp. 19596-19605, 2018.

[42] Z. Zhang and M. Qiu, Opt. Express, vol. 12, no. 17, pp. 3988-3995, 2004.

[43] Y. Yu, H. Hu, L. K. Oxenlowe, K. Yvind, and J. Mork, Opt. Lett., vol. 40, no. 10, p. $2357,2015$.

[44] Y. Yu, M. Heuck, H. Hu, W. Xue, C. Peucheret, Y. Chen, L. K. Oxenløwe, K. Yvind, and J. Mørk, Appl. Phys. Lett., vol. 105, no. 6, 2014.

[45] G. Moille, S. Combrié, and A. De Rossi, Phys. Rev. A, vol. 94, no. 2, p. 23814, Aug. 2016.

[46] B. R. Bennett, R. A. Soref, and J. A. Del Alamo, IEEE J. Quantum Electron., vol. 26, no. 1 , pp. 113-122, 1990.

[47] H. Kawashima, Y. Tanaka, N. Ikeda, Y. Sugimoto, T. Hasama, and H. Ishikawa, IEEE J. Quantum Electron., vol. 44, no. 9, pp. 841-849, 2008.

[48] P. E. Barclay, K. Srinivasan, and O. Painter, vol. 13, no. 3, pp. 801-820, 2005.

[49] F. G. Della Corte, G. Cocorullo, M. Iodice, and I. Rendina, Appl. Phys. Lett., vol. 77, no. 11, pp. 1614-1616, 2000.

[50] D. A. Bekele, Y. Yu, H. Hu, P. Guan, L. Ottaviano, M. Galili, L. K. Oxenløwe, K. Yvind, and J. Mork, Opt. Lett., vol. 43, no. 4, p. 955, Feb. 2018.

[51] S. M. Sze and K. K. Ng, Physics of semiconductor devices, John wiley \& sons, 2006.

[52] Y. Rosenwaks, Y. Shapira, and D. Huppert, Phys. Rev. B, vol. 45, no. 16, pp. $9108-$ 9119, Apr. 1992.

[53] L. E. Black, in New Perspectives on Surface Passivation: Understanding the Si-Al2O3 Interface, Springer, 2016, pp. 15-28.

[54] M. Heuck, S. Combrié, G. Lehoucq, S. Malaguti, G. Bellanca, S. Trillo, P. T. 


\section{WILEY-VCH}

Kristensen, J. Mørk, J. P. Reithmaier, and A. De Rossi, Appl. Phys. Lett., vol. 103, no. $18,2013$.

[55] D. Breuer and K. Petermann, IEEE Photonics Technol. Lett., vol. 9, no. 3, pp. 398-400, 1997.

[56] D. A. B. Miller, Proc. IEEE, vol. 97, no. 7, pp. 1166-1185, 2009.

[57] X. Zhao, C. Lou, H. Zhou, D. Lu, and L. Huo, Opt. Express, vol. 18, no. 23, p. 23657, 2010.

[58] O. Leclerc, B. Lavigne, E. Balmefrezol, P. Brindel, L. Pierre, D. Rouvillain, and F. Seguineau, J. Light. Technol., vol. 21, no. 11, pp. 2779-2790, 2003.

[59] R. Slavík et al., Nat. Photon., vol. 4, no. 10, pp. 690-695, 2010.

[60] G. P. Agrawal, Fiber-optic communication systems, vol. 222. John Wiley \& Sons, 2012.

[61] A. E. Willner, S. Khaleghi, M. R. Chitgarha, and O. F. Yilmaz, J. Light. Technol., vol. 32, no. 4, pp. 660-680, 2014.

[62] F. Öhman, S. Bischoff, B. Tromborg, and J. Mørk, IEEE J. Quantum Electron., vol. 40, no. 3 , pp. $245-255,2004$.

[63] D. A. Bekele, Y. Yu, H. Hu, Y. Ding, A. Sakanas, L. Ottaviano, E. Semenova, L. K. Oxenløwe, K. Yvind, and J. Mork, in Proceedings of SPIE, 2017, vol. 10345.

[64] D. A. Bekele, Y. Yu, H. Hu, P.-Y. Bony, L. Ottaviano, L. K. Oxenlowe, K. Yvind, and J. Mork, Presented at 2017 CLEO/Europe-EQEC, Munich, Germany, June 2017, pp. 11. https://doi.org/10.1109/CLEOE-EQEC.2017.8087107

[65] H. Murai, M. Kagawa, H. Tsuji, and K. Fujii, IEEE J. Sel. Top. Quantum Electron., vol. 13, no. 1, pp. 70-78, 2007.

[66] C. Koos, P. Vorreau, T. Vallaitis, P. Dumon, W. Bogaerts, R. Baets, B. Esembeson, I. Biaggio, T. Michinobu, F. Diederich, W. Freude, and J. Leuthold, Nat. Photon., vol. 3, no. 4, pp. 216-219, 2009.

[67] K. Igarashi, K. Katoh, and K. Kikuchi, Opt. Express, vol. 15, no. 3, pp. 845-851, 2007.

[68] K. Lenglé, T. N. Nguyen, M. Gay, L. Bramerie, J.-C. Simon, A. Bazin, F. Raineri, and R. Raj, Opt. Lett., vol. 39, no. 8, pp. 2298-2301, 2014.

[69] I. Cestier, a Willinger, V. Eckhouse, G. Eisenstein, S. Combrié, P. Colman, G. Lehoucq, and a De Rossi, Opt. Express, vol. 19, no. 7, pp. 6093-6099, 2011.

[70] J. S. Pelc, K. Rivoire, S. Vo, C. Santori, D. A. Fattal, and R. G. Beausoleil, Opt. 


\section{WILEY-VCH}

Express, vol. 22, no. 4, pp. 3797-3810, 2014.

[71] G.-R. Lin, S.-P. Su, C.-L. Wu, Y.-H. Lin, B.-J. Huang, H.-Y. Wang, C.-T. Tsai, C.-I. Wu, and Y.-C. Chi, Sci. Rep., vol. 5, p. 9611, 2015.

[72] Z. Yu and S. Fan, Nat. Photon., vol. 3, no. 2, p. 91, 2009.

[73] Z. Wang, Y. Chong, J. D. Joannopoulos, and M. Soljačić, Nature, vol. 461, no. 7265, p. 772, 2009.

[74] B. Peng, Ş. K. Özdemir, F. Lei, F. Monifi, M. Gianfreda, G. L. Long, S. Fan, F. Nori, C. M. Bender, and L. Yang, Nat. Phys., vol. 10, no. 5, p. 394, 2014.

[75] L. Fan, J. Wang, L. T. Varghese, H. Shen, B. Niu, Y. Xuan, A. M. Weiner, and M. Qi, Science (80-. )., vol. 335, no. 6067, pp. 447-450, 2012.

[76] Y. Xu and A. E. Miroshnichenko, Phys. Rev. B, vol. 89, no. 13, p. 134306, 2014.

[77] Y. Yu, Y. Chen, H. Hu, W. Xue, K. Yvind, and J. Mork, Laser \& Photon. Rev., vol. 9, no. 2, pp. 241-247, 2015.

[78] Y. Yu, W. Xue, E. Semenova, K. Yvind, and J. Mork, Nat. Photon., vol. 11, no. 2, pp. 81-84, 2017.

[79] J. Mork, Y. Chen, and M. Heuck, Phys. Rev. Lett., vol. 113, no. 16, p. 163901, 2014.

[80] T. S. Rasmussen, Y. Yu, and J. Mork, Laser Photon. Rev., vol. 11, no. 5, p. 1700089 , 2017.

[81] T. S. Rasmussen, Y. Yu, and J. Mork, Opt. Express, vol. 26, no. 13, pp. 16365-16376, 2018.

[82] A. R. Zali, M. K. Moravvej-Farshi, Y. Yu, and J. Mork, J. Light. Technol., vol. 36, no. 23, pp. 5611-5616, 2018.

[83] J. Mork, Y. Yu, T. S. Rasmussen, E. Semenova, and K. Yvind, IEEE J. Sel. Top. Quantum Electron., vol. 25, no. 6, pp. 1-14, 2019.

[84] G. Moille, S. Combrié, K. Fuchs, M. Yacob, J. P. Reithmaier, and A. de Rossi, Opt. Lett., vol. 42, no. 4, pp. 795-798, 2017.

[85] Y. Ding, H. Hu, H. Ou, L. K. Oxenløwe, and K. Yvind, presented at 2015 Conf. Lasers Electro-Optics (CLEO), San Jose, CA, May 2015.

[86] T. Tanabe, H. Taniyama, and M. Notomi, J. Light. Technol., vol. 26, no. 11, pp. 1396$1403,2008$.

[87] L.-D. Haret, X. Checoury, F. Bayle, N. Cazier, P. Boucaud, S. Combrié, and A. de 


\section{WILEY-VCH}

Rossi, Opt. Express, vol. 21, no. 8, pp. 10324-10334, 2013.

[88] J. Leuthold, D. M. Marom, S. Cabot, J. J. Jaques, R. Ryf, and C. R. Giles, J. Light. Technol., vol. 22, no. 1, p. 186, 2004.

[89] T. Tanabe, K. Nishiguchi, E. Kuramochi, and M. Notomi, Opt. Express, vol. 17, no. 25, pp. 22505-22513, 2009.

[90] T. Tanabe, H. Sumikura, H. Taniyama, A. Shinya, and M. Notomi, Appl. Phys. Lett., vol. 96, no. 10, p. 101103, 2010.

[91] F. Zangeneh-Nejad and R. Fleury, Phys. Rev. Lett., vol. 122, no. 1, p. 14301, 2019. 